\title{
Über die biologischen Wechselbeziehungen zwischen Fett, Vitamin A und Vitamin B=Faktoren.
}

\author{
Von \\ Shunyichi Yonechi. \\ (米 地 俊 一) \\ (Aus der Medizinischen Klinik von Prof. Dr. T. Kumagai, \\ Kaiserl. Tohoku-Universität zu Sendai.)
}

\section{Einleitung.}

Die Vitaminforschung ist schon längt aus den Kindes'schuhen und geht jetzt mit mächtigen Schritten voran. Heute, da die biochemische oder biologische Bedeutung der einzelnen Vitamine allmählich klar gestellt worden ist, besteht die Aussicht, einerseits die Vitamine chemisch zu analysieren oder zu synthetisieren, andererseits sind viele Forschungsergebnisse über ihren Synergismus mit oder ihren Antagonismus gegen verschiedene innersekretorische Drüsen bei Eiweiss-, Kohlehydrat- und Fettstoffwechsel oder über die Wechselbeziehungen $z$ wischen den einzelnen Vitaminen veröffentlicht worden. Im Jahre 1929 haben Evans und Lepkovsky ${ }^{1)}$ einen Bericht ,Sparing Action of Fat on antineuritic Vitamin B" mitgeteilt und dann im Jahre 19322) sich dahin geäussert, dass Fettarten, Schmelzpunkt und Unsättigungsgrad keinen Einfluss auf die ,sparing action of fat" haben, nur dass sich bei solchem Fett mit hohem Schmelzpunkt, d. h. mit niedrigem Resorptionsgrad wie z.B. bei Stearinsäure, keine ,sparing action" geltend macht. Ferner berichteten diese Autoren, ${ }^{3)}$ dass bei Anwendung von Fettsäure und ihren Verbindungen bei solchen mit hohem Resorptionsgrad wie Caprinsäure, Capririnsäure u.a. günstige Ergebnisse erzielt. Darauf teilten $\operatorname{sie}^{4}$ mit, dass antineurales Vitamin B sowohl per os als auch intraperitoneal wirksam ist. Wichtig ist dieses Problem für die neuerdings bei der Verhütung sowie der Heilung verschiedener Krankheiten besonders beachtete Diättberapie und Ernährungslehre. Es gibt dabei noch viele Fragen, die noch weiterer Forschung bedürfen. Dies alles hat mich veranlasst, zuerst Evans ,sparing action of fat", nachzuprufen. 


\section{Erster Teil.}

Versuche mit einigen Fettarten und Vitamin B-Faktoren.

I. Fütterung von Tauben mit Baumwollsamenöl.

Evans u. Lepkovsky haben als Material junge Ratten verwendet und auf Grund der eingetretenen $B_{1}$-Avitaminoseerscheinungen, der Körpergewichtszunahme, der Lebensdauer u. a. ihr Urteil abgegeben, doch als Material sind m. E. erwachsene Ratten darum den jungen überlegen, weil selbst bei einer Gruppe aus denselben Würfen unleugbar sowohl das Wachstum wie auch der Prozentsatz der Erkrankung verschieden sein können. Zur ersten Versuchsreihe verwendete ich aber Tauben, weil ich sie mir leichter verschaffen konnte und sie leichter an B-Avitaminose erkranken. Das Futter bestand für einen Tag aus :

1) poliertem Reis.....etwa $30 \mathrm{~g}$ (ad libitum),

2) Vitamin B-Komplex...... Oryzanin fortius $0,03 \mathrm{~g}$, ,Sankyo",

3) thermostabilem Vitamin $B\left(B_{0}\right)$, der im Autoklar bei $120^{\circ} \mathrm{C} 4$ Stunden lang vorbehandelten Wasserlösung des Oryzanin fortius "Sankyo",

4) Baum wollsamenöl (a)...... Wesson Oil (Wesson Oil Co. U.S.A.) (Zwangsfütterung mittels einer Schlundsonde $10 \mathrm{ccm}$ pro Tag) und

5) Baumwollsamenöl (b), dem in gleicher Weise wie bei Oryzanin fortius im Autoklav vorbehandelten Wesson Oil.

Jede Gruppe bestand aus 3 Tauben. Obiges Futtermaterial lässt sich verschieden zusammenstellen, wie Tabelle I zeigt.

Bei dem Versuch wurde besonders auf die $\mathrm{Zu}$ - und Abnahme des Körpergewichts, das Auftreten $\mathrm{B}_{1}$-avitaminotischer Symptome, die Lebensdauer und die sog. toxische Erscheinung von Fett geachtet. Die schädliche Wirkung von übermässiger Fettfütterung besteht bekanntlich in Abmagerung, Verklebung und Büschelung von Haaren mit später einsetzendem Ausfall u. a., worauf weiterhin noch genauer eingegangen werden wird. Die Versuchsergebnisse findet man in der folgenden Tabelle I anschaulich zusammengestellt.

\section{Zu- und Abnahme des Körpergewichts.}

Sowohl bei den nur mit poliertem Reis als auch bei den mit Reis und Öl gefütterten Gruppen ruft Zusetzen des Vitamin B-Komplexes oder des thermostabilen Vitamins B auf die Zu- und Abnahme des Körpergewichts fast gar keinen Einfluss hervor. 
Weehselbeziehungen zwischen Fett, Vitamin A $\mathfrak{u}$. Vitamin B-Faktoren

Tabelle $\mathrm{I}$.

Fütterungsversuch von Tauben mit Baumwollsamenöl.

\begin{tabular}{|c|c|c|c|c|c|c|c|c|}
\hline \multicolumn{2}{|c|}{ Gruppe } & I & II & III & IV & V & VI & VII \\
\hline $\begin{array}{c}\text { Bestandteile } \\
\text { des Futters } \\
(\%)\end{array}$ & $\begin{array}{l}\text { Eiweiss } \\
\text { Kohlehydrat } \\
\text { Fett }\end{array}$ & $\begin{array}{r}5,15 \\
94,07 \\
0,78\end{array}$ & $\begin{array}{r}5.15 \\
94,07 \\
0,78\end{array}$ & $\begin{array}{r}5,15 \\
94,07 \\
0,78\end{array}$ & $\begin{array}{r}3,6 \\
67,5 \\
28,0 \\
(a) \\
\end{array}$ & $\begin{array}{r}3,6 \\
67,5 \\
28,0 \\
\text { (b) }\end{array}$ & $\begin{array}{r}3,6 \\
67,5 \\
28,0 \\
(a) \\
\end{array}$ & $\begin{array}{r}3,6 \\
67,5 \\
28,0 \\
(\mathrm{a}) \\
\end{array}$ \\
\hline $\begin{array}{c}\text { Vitamin B } \\
\text { Faktor } \\
\text { (g) }\end{array}$ & $\begin{array}{l}\text { Thermostabiles } \\
\text { Vitamin B } \\
\text { Vitamin B Kom- } \\
\text { plex }\end{array}$ & & 0,03 & 0,03 & & & 0,03 & 0,03 \\
\hline $\begin{array}{c}\text { Körpergewichts- } \\
\text { zunah me } \\
(\%)\end{array}$ & $\begin{array}{l}\text { Maximum } \\
\text { Minimum } \\
\text { Mittelwert }\end{array}$ & $\begin{array}{r}20,7 \\
7,0 \\
12,7\end{array}$ & $\begin{array}{r}12,5 \\
7,1 \\
9,5\end{array}$ & $\begin{array}{l}16,0 \\
10,7 \\
14,0\end{array}$ & $\begin{array}{l}25,0 \\
10,0 \\
17,6\end{array}$ & $\begin{array}{r}11,1 \\
5,7 \\
8,7\end{array}$ & $\begin{array}{l}41,6 \\
35,0 \\
38,3\end{array}$ & $\begin{array}{l}13,9 \\
10,3 \\
12,6\end{array}$ \\
\hline $\begin{array}{c}\text { Körpergewichts } \\
\text { abnahme } \\
(\%)\end{array}$ & $\begin{array}{l}\text { Maximum } \\
\text { Minimum } \\
\text { Mittelwert }\end{array}$ & $\begin{array}{l}32,1 \\
25,0 \\
28,2\end{array}$ & $\begin{array}{l}29,6 \\
21,8 \\
26,6\end{array}$ & $\begin{array}{l}24,0 \\
19,2 \\
21,5\end{array}$ & $\begin{array}{c}20,0 \\
0 \\
9,5\end{array}$ & $\begin{array}{l}42,8 \\
21,9 \\
30,2\end{array}$ & $\begin{array}{r}20,0 \\
8,2 \\
14,1\end{array}$ & $\begin{array}{l}31,0 \\
24,1 \\
19,8\end{array}$ \\
\hline $\begin{array}{c}\text { Entstehungszeit } \\
\text { der } B_{1}-A \text { vita- } \\
\text { minose } \\
\text { (Tag) }\end{array}$ & $\begin{array}{l}\text { Maximum } \\
\text { Minimum } \\
\text { Mittelwert }\end{array}$ & $\begin{array}{l}25 \\
20 \\
22,6\end{array}$ & $\begin{array}{l}37 \\
29 \\
31,6\end{array}$ & $\mathbf{X}$ & $\begin{array}{l}25 \\
18 \\
21,0\end{array}$ & $\begin{array}{l}33 \\
26 \\
29,5\end{array}$ & $\begin{array}{l}22 \\
18 \\
20,0\end{array}$ & $\mathrm{x}$ \\
\hline $\begin{array}{l}\text { Schädigungser- } \\
\text { scheinung über- } \\
\text { mässigen Fetts } \\
\text { (Tag) }\end{array}$ & $\begin{array}{l}\text { Maximum } \\
\text { Minimum } \\
\text { Mittelwert }\end{array}$ & $\mathrm{K}$ & $\mathbf{K}$ & K & $\begin{array}{l}14 \\
6 \\
8,6\end{array}$ & $\begin{array}{l}9 \\
5 \\
7,0\end{array}$ & $\begin{array}{l}16 \\
13 \\
14,5\end{array}$ & $\begin{array}{l}16 \\
15 \\
15,5\end{array}$ \\
\hline $\begin{array}{l}\text { Lebensdauer } \\
\text { (Tag) }\end{array}$ & $\begin{array}{l}\text { Maximum } \\
\text { Minimum } \\
\text { Mittelwert }\end{array}$ & $\begin{array}{l}34 \\
24 \\
29,3\end{array}$ & $\begin{array}{l}40 \\
34 \\
36,6\end{array}$ & $\begin{array}{l}\S \\
\S\end{array}$ & $\begin{array}{l}30 \\
11 \\
18,3\end{array}$ & $\begin{array}{l}40 \\
14 \\
28,0\end{array}$ & $\begin{array}{l}25 \\
21 \\
23,0\end{array}$ & $\begin{array}{l}33 \\
28 \\
30,5\end{array}$ \\
\hline
\end{tabular}

Anm.X......Keine $B_{1}$-Avitaminose.

K.......Keine Schädigungserscheinung.

(a)......Wesson Oil.

(b).......im Autoklav vorbehandelten Wesson 0 il.

$\S$.......die ganze Versuchszeit hindurch am Leben geblieben.

Auch zwischen den mit und den ohne Öl gefütterten Gruppen ist kaum ein Unterschied nachweisbar.

2. Entstehung der $\mathrm{B}_{1}$-Avitaminose (Beriberisymptome).

Beim Vergleich der mit Öl gefütterten Gruppen mit denen ohne Öl gefütterten kann man erkennen, dass das Öl niemals eine sparende, sondern umgekehrt eine $B_{1}$-Avitaminose schwach fördernde Wirkung ausübt. 
3. Schädigende Wirkung übermässigen Fetts.

Hier kann man beobachten, dass der Vitamin B-Komplex sowie thermostabiles Vitamin $B\left(d\right.$. h. Vitamin $B_{\mathrm{q}}$ ) die schädigende Wirkung übermässigen Fetts veřögert oder hemmt.

\section{Lebensdauer.}

Bei den mit Öl gefütterten Gruppen ist die Lebensdauer im allgemeinen kurz, bei Zusatz des Vitamin B-Komplexes etwas länger, während thermostabiles Vitamin B keinen Einfluss darauf hat. Auch bei den ohne Öl gefütterten Gruppen verlängert Vitamin $\mathrm{B}_{2}$ die Lebensdauer nicht.

Wie aus Obigem ersichtlich, lässt sich Evans u. Lepkorskys „sparing action of fat on antineuritic Vitamin B" nicht nachweisen, sondern umgekehrt eine schädliche Wirkung übermässigen Baumwollsamenöls und eine hemmende Wirkung des Vitamin B-Komplexes sowie des Vitamins $\mathrm{B}_{2}$ auf die Schädigung durch Öl.

\section{Fütterung von Ratten mit Baumwollsamenöl.}

Man könnte hier vielleicht einwenden, ich hätte beim Versuche mit Tauben Evans' u. Lepkovskys ,;sparing action" nicht nachweisen können, weil die Taube ein Pflanzenfresser ist und deshalb nicht viel Fett zu ertragen vermag. Deshalb habe ich nun in einer zweiten Versuchsreihe erwachsene Ratten benutzt.

Das Futter bestand aus :

1) Casëin 20\%,

2) Reismehl 70\%,

3) Zucker $4 \%$, und

4) einem Salzgemisch 4\% (nach MacCollu m${ }^{5}$ )

$\begin{array}{lc}\text { (Natr. chloratum } & \mathbf{5 1 , 0} \\ \text { Mag. sulphuricum (Krystall) } & \mathbf{1 5 9 , 0} \\ \text { Natr. phosphoricum (monobasisches) } & \mathbf{1 0 4 , 1} \\ \text { Calc. phosphoricum (monobasisches) } & 162,0 \\ \text { Kal. phosphoricum (dibasisches) } & \mathbf{2 8 6 , 2} \\ \text { Ferr. citricum } & \mathbf{3 5 , 4} \text { und } \\ \text { Calc. lacticum } & 390,0 \text { ), }\end{array}$

Die Prozentsätze des Futters bestanden aus Eiweiss $\quad 24,57 \%$ Kohlehydrat $\quad \mathbf{7 4 , 7} \%$ und Fett $0,63 \%$,

(Anm. Die Prozentsätze von Eiweiss, Kohlehydrat und Fett der Nahrung bei den Versuch sind nach $\mathrm{Sa}$ ik is ,The Chemical Analysis of Food in Japan,6)“ Tokyo 1931 berechnet.) 
und aus dem Vitamin B-Komplex......Oryzanin fortius „Sankyo“, wie beim Versuch mit Tauben.

Jede Gruppe bestand aus 5 Ratten. Dem Grundfutter wurden Vitamin B-Komplex, thermostabiles Vitamin B und Baumwollsamenöl je nach dem Zweck zugesetzt und das Grundfutter von $30 \mathrm{~g}$ für einen Tag ad libitum gegeben.

Die Versuchsergebnisse findet man in Tabelle II zusammengestellt.

Tabelle II.

Fütterungsversuch der Ratten mit Baumwollsamenöl.

\begin{tabular}{|c|c|c|c|c|c|c|c|c|c|c|}
\hline \multicolumn{2}{|c|}{ Gruppe } & I & II & III & IV & V & VI & VII & VIII & IX \\
\hline $\begin{array}{c}\text { Bestandteile } \\
\text { des Futter's } \\
(\%)\end{array}$ & $\begin{array}{l}\text { Eiweiss } \\
\text { Kohlehydrat } \\
\text { Fett }\end{array}$ & $\begin{array}{c}24,57 \\
74,7 \\
0,63\end{array}$ & $\begin{array}{c}24,57 \\
74,7 \\
0,68\end{array}$ & $\begin{array}{c}24,57 \\
74,7 \\
0,63\end{array}$ & $\begin{array}{c}22,3 \\
67,9 \\
10,0 \\
\text { (a) }\end{array}$ & $\begin{array}{c}18,9 \\
58,2 \\
23,5 \\
(a)\end{array}$ & $\begin{array}{c}18,9 \\
58,2 \\
23,5 \\
\text { (b) }\end{array}$ & $\begin{array}{r}18,9 \\
58,2 \\
23,5 \\
(\mathrm{a})\end{array}$ & $\begin{array}{r}18,9 \\
58,2 \\
23,5 \\
\text { (a) } \\
\end{array}$ & $\begin{array}{r}16,4 \\
33,6 \\
50,0 \\
(\text { a) } \\
\end{array}$ \\
\hline $\begin{array}{c}\text { Vitamin B } \\
\text { Faktor } \\
\text { (g) }\end{array}$ & $\begin{array}{l}\text { Thermostabil } \\
\text { Komplex }\end{array}$ & & 0,03 & 0,03 & & & & 0,03 & 0,03 & 0,03 \\
\hline $\begin{array}{c}\text { Körperge- } \\
\text { wichtszu- } \\
\text { nahme } \\
(\%)\end{array}$ & $\begin{array}{l}\text { Maximum } \\
\text { Minimum } \\
\text { Mittelwert }\end{array}$ & $\begin{array}{l}19,2 \\
17,9 \\
18,5\end{array}$ & $\begin{array}{l}25,0 \\
13,0 \\
19,1\end{array}$ & $\begin{array}{l}22,2 \\
11,8 \\
19,8\end{array}$ & $\begin{array}{l}23,1 \\
12,5 \\
17,8\end{array}$ & $\begin{array}{r}14,3 \\
5,2 \\
9,9\end{array}$ & $\begin{array}{l}20,8 \\
18,2 \\
19,1\end{array}$ & $\begin{array}{r}13,6 \\
5,0 \\
9,7\end{array}$ & $\begin{array}{r}15,4 \\
5,5 \\
8,3\end{array}$ & $\begin{array}{l}0 \\
0 \\
0\end{array}$ \\
\hline $\begin{array}{c}\text { Körperge- } \\
\text { wichtsab- } \\
\text { nahme } \\
(\%)\end{array}$ & $\begin{array}{l}\text { Maximum } \\
\text { Minimum } \\
\text { Mittelwert }\end{array}$ & $\begin{array}{l}7,7 \\
3,5 \\
4,9\end{array}$ & $\begin{array}{l}0 \\
0 \\
0\end{array}$ & $\begin{array}{l}5,9 \\
0 \\
1,2\end{array}$ & $\begin{array}{l}19,2 \\
15,6 \\
17,4\end{array}$ & $\begin{array}{l}43,5 \\
21,0 \\
37,4\end{array}$ & $\begin{array}{l}33,3 \\
31,8 \\
32,3\end{array}$ & $\begin{array}{r}35,0 \\
9,0 \\
22,6\end{array}$ & $\begin{array}{c}28,5 \\
0 \\
14,7\end{array}$ & $\begin{array}{l}50,0 \\
12,5 \\
26,6\end{array}$ \\
\hline $\begin{array}{c}\text { Entstehungs- } \\
\text { zeit der } B_{1}- \\
\text { Avitaminose } \\
\text { (Tag) }\end{array}$ & $\begin{array}{l}\text { Maximum } \\
\text { Minimum } \\
\text { Mittelwert }\end{array}$ & $\begin{array}{l}64 \\
61 \\
62,3\end{array}$ & $\begin{array}{l}72 \\
64 \\
68,2\end{array}$ & $\mathrm{X}$ & $\begin{array}{l}63 \\
58 \\
60,0\end{array}$ & $\begin{array}{l}X(2) \\
46 \\
44,5\end{array}$ & $\begin{array}{l}60 \\
53 \\
\\
57,0\end{array}$ & $\begin{array}{l}53 \\
35 \\
45,5\end{array}$ & \begin{tabular}{|c|}
$\mathrm{X}$ \\
2 \\
$(2) ?$ \\
62 \\
$(2) ?$
\end{tabular} & $\begin{array}{l}21 \\
18 \\
19,5\end{array}$ \\
\hline $\begin{array}{l}\text { Schädigungs- } \\
\text { erscheinung } \\
\text { ubbermässi- } \\
\text { gen Fetts } \\
\text { (Tag) }\end{array}$ & $\begin{array}{l}\text { Maximum } \\
\text { Minimum } \\
\text { Mittelwert }\end{array}$ & $\mathbf{K}$ & $\mathbf{K}$ & $\mathbf{K}$ & $\begin{array}{l}57 \\
50 \\
53,5\end{array}$ & $\begin{array}{l}29 \\
22 \\
25,4\end{array}$ & $\begin{array}{l}35 \\
34 \\
34,6\end{array}$ & $\begin{array}{l}38 \\
25 \\
33,0\end{array}$ & $\begin{array}{l}46 \\
27 \\
38,0\end{array}$ & $\begin{array}{l}10 \\
7 \\
8,6\end{array}$ \\
\hline $\begin{array}{l}\text { Lebensdauer } \\
\text { (Tag) }\end{array}$ & $\begin{array}{l}\text { Maximnm } \\
\text { Minimum } \\
\text { Mittelwert }\end{array}$ & $\begin{array}{l}\$ \\
\$\end{array}$ & $\begin{array}{l}\$ \\
\S\end{array}$ & $\begin{array}{l}\S \\
\S\end{array}$ & $\begin{array}{l}\S \\
\S\end{array}$ & $\begin{array}{l}59 \\
26 \\
45,0\end{array}$ & $\begin{array}{l}78 \\
76 \\
77,0\end{array}$ & $\mid \begin{array}{c}\$(1) \\
44 \\
52,0 \\
\S(1)\end{array}$ & $\begin{array}{c}\$(2) \\
49 \\
58,3 \\
\$(2)\end{array}$ & $\begin{array}{l}30 \\
17 \\
24,5\end{array}$ \\
\hline
\end{tabular}

Anm. X.......Keine $B_{1}$-A vitaminose.

K.......Keine Schädigungserscheinung übermässigen Fetts beobachtet.

8....... Die ganze Versuchzeit hindurch am Leben geblieben.

( )......Zahl des Versuchstiers.

(a)....... Wesson Oil.

(b)...... Im Autoklav vorbehandeltes Wesson $0 i l$. 
1. Zu- und Abnahme des Körpergewichts.

Bei den Gruppen ohne Baumwollsamenöl übt der Vitamin B-Komplex sowie das thermostabile Vitamin B keinen besonderen Einfluss auf das Körpergewicht aus.

Bei den Gruppen mit $10 \%$ und etwa $24 \%$ Öl fördert zwar der Zusatz des Vitamin B-Komplexes sowie des thermostabilen Vitamin B die Zunahme des Gewichts, aber zwischen diesen beiden Gruppe findet man keinen deutlichen Unterschied. Bei der Gruppe mit 50\% Öl ist keine Wirkung des Vitamins zu finden.

Was die Körpergewichtsabnahme betrifft, so ist die Abmagerung wohl bei Zusatz des Vitamin B-Komplexes sowie des thermostabilen Vitamins B geringfügig, aber je mehr Öl man gibt, umso stärker tritt sie auf. Im allgemeinen zeigt sich bei den Gruppen mit Öl auffallende Abmagerung.

2. Entstehung der $\mathbf{B}_{1}$-Avitaminose.

Je grösser die Menge des Baumwollsamenöls, desto früher tritt $\mathrm{B}_{1}$-A vitaminose auf, wobei thermostabiles Vitamin B unwirksam ist. Infolge auftretender toxischer Erscheinungen durch übermässiges Fett zeigen sich trotz Anwesenheit des Vitamin B-Komplexes sogar beriberiähnliche Symptome, z. B. Gangstörung. Also steht unserVersuch mit dem Ergebnis von Evans u. Lepk ov sky nicht im Einklang.

3. Schädigende Wirkung übermässigen Fetts.

Je grösser die Ölmenge, desto früher und schneller entstehen und verlaufen die Schädigungen durch Fett, wobei der Vitamin B-Komplex sowie das thermostabile Vitamin B die Schädigung durch Fett gar nicht hemmen. (S. Abb. 1.)

\section{Lebensdauer.}

Unter den Gruppen mit Öl blieb nur die Gruppe mit 10\% Öl die ganze Versuchszeit hindurch am Leben, während sonst der Tod umso früher eintrat, je grösser die Ölmenge war. Auf die Lebensdauer haben der Vitamin B-Komplex sowie thermostabiles Vitamin B keinen Einfluss.

Wie bei Tauben, so ist auch bei Ratten keine ,,sparing action of fat on antineuritic Vitamin B", sondern umgekehrt eine $B_{1}$-Avitaminose fördernde Wirkung des Öls festzustellen, wobei gefunden wurde, 


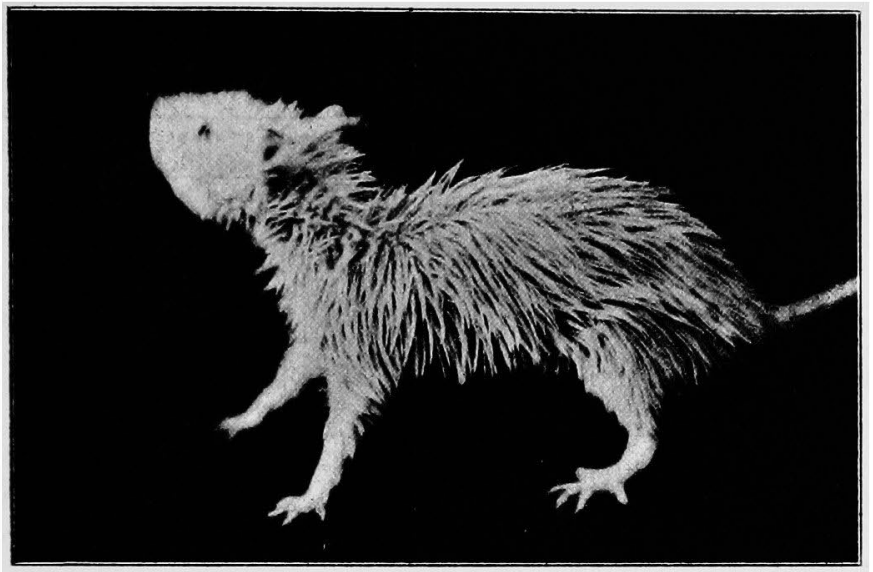

Abb. 1. Das Grundfutter mit 50\% Baumwollsamenöl und Vitamin B-Komplex gegeben. (S. Tabelle I, Gruppe VII.) Aufgenommen am 27 Versuchstage.

dass übermässige Fettgabe im umgekehrten Verhältnis zur Ölmenge schädigend wirkt. Zuerst werden Gefieder oder Haare beschmiert, haften zusammen und werden büschelartig. Dann treten Haarausfall, ödematöse Anschwellung der Extremitäten, seborrhoeaartige Hautveränderungen, meist an blossliegenden Teilen, Abmagerung und Atrophie ein; dadurch kommt der Tod auffallend früh.

\section{Fütterung von Ratten mit Lebertran} und Butter.

Bei den obigen zwei Versuchsreihen ist ein vegetabilisches $\ddot{O} 1$ gebraucht worden. Ich fragte mich nun, ob animalisches Fett oder Ö̉ jene „sparing action" bewirken könne. Deshalb sind bei dieser Versuchsreihe feste Butter von hohem Schmelzpunkt, öliger Lebertran von niedrigem Schmelzpunkt und als Vitamin B-Quelle getrokknete Hefe verwandt worden. Nach der Angabe von Evans u. Lepkovsky, dass bei einer Fütterung mit Kohlehydraten als Grundnahrung sehr klar eine hochgradige ,sparing action" zu beobachten sei, habe ich hier Casëin und Reismehl in einem anderen Verhältnis zusammengestellt.

Jede Gruppe bestand aus 4 Ratten, wobei die mit Lebertran gefütterten Gruppen auch dem Körpergewicht nach eingeteilt wurden.

Die Zusammensetzung der Nahrung und die Versuchsergebnisse gibt Tabelle III wieder. 
Tabelle

Fütterungsversuch von Ratten

\begin{tabular}{|c|c|c|c|c|c|c|c|c|c|c|}
\hline \multicolumn{2}{|c|}{ Gruppe } & I & II & III & IV & $\nabla$ & VI & VII & VIII & IX \\
\hline \multicolumn{2}{|c|}{$\begin{array}{l}\text { Körpergewicht } \\
(\mathrm{g})\end{array}$} & $200>$ & $200>$ & $200>$ & $200>$ & $200>$ & $200>$ & $\begin{array}{r}100- \\
140\end{array}$ & $\begin{array}{r}150- \\
190\end{array}$ & $200>$ \\
\hline $\begin{array}{c}\text { Bestandteile } \\
\text { des Futters } \\
(\%)\end{array}$ & $\begin{array}{l}\text { Eiweiss } \\
\text { Kohlehydrat } \\
=\left\{\begin{array}{l}\text { Butter } \\
\text { Lebertran }\end{array}\right.\end{array}$ & $\begin{array}{l}34,09 \\
55,17 \\
10,74\end{array}$ & $\begin{array}{l}34,09 \\
55,17 \\
10,74\end{array}$ & $\begin{array}{l}22,87 \\
43,91 \\
31,49\end{array}$ & $\begin{array}{l}22,87 \\
43,91 \\
31,49\end{array}$ & $\begin{array}{l}17,16 \\
31,94 \\
50,16\end{array}$ & $\begin{array}{l}17,16 \\
31,94 \\
50,16\end{array}$ & $\begin{array}{l}34,09 \\
55,17 \\
10,74\end{array}$ & $\begin{array}{l}34,09 \\
55,17 \\
10,74\end{array}$ & $\begin{array}{l}34,09 \\
55,17 \\
10,74\end{array}$ \\
\hline Vitamin & $\begin{array}{l}\text { Vitamin A } \\
\text { Vitamin B Kom- } \\
\text { plex (Hefe) }(g)\end{array}$ & $\begin{array}{r}7,4 \\
\text { R.E. } \\
0,2\end{array}$ & $\begin{array}{r}7,4 \\
\text { R.E. }\end{array}$ & $\begin{array}{c}22,1 \\
\text { R.E. } \\
0,2\end{array}$ & $\begin{array}{l}22,1 \\
\mathrm{R} . \mathrm{E} .\end{array}$ & $\begin{array}{c}33,8 \\
\mathrm{R}, \mathrm{E} . \\
0,2\end{array}$ & $\begin{array}{l}33,8 \\
\text { R.E. }\end{array}$ & $\begin{array}{r}183,8 \\
\text { R.E. } \\
0,2\end{array}$ & $\begin{array}{r}183,8 \\
\text { R.E. } \\
0,2\end{array}$ & $\begin{array}{c}183,8 \\
\text { R.E. } \\
0,2\end{array}$ \\
\hline $\begin{array}{c}\text { Körperge- } \\
\text { wichtszu- } \\
\text { nahme } \\
(\%)\end{array}$ & $\begin{array}{l}\text { Maximum } \\
\text { Minimum } \\
\text { Mittelwert }\end{array}$ & $\begin{array}{l}4,5 \\
0 \\
1,5\end{array}$ & $\begin{array}{l}0 \\
0 \\
0\end{array}$ & $\begin{array}{l}4,5 \\
0 \\
2,9\end{array}$ & $\begin{array}{l}3,8 \\
0 \\
2,4\end{array}$ & $\begin{array}{l}29,3 \\
16,7 \\
22,6\end{array}$ & $\begin{array}{l}0 \\
0 \\
0\end{array}$ & $\begin{array}{l}36,3 \\
33,3 \\
34,8\end{array}$ & $\begin{array}{l}23,5 \\
15,8 \\
19,3\end{array}$ & $\begin{array}{l}4,7 \\
0 \\
2,4\end{array}$ \\
\hline $\begin{array}{c}\text { Körperge- } \\
\text { wiehtsab- } \\
\text { nahme } \\
(\%)\end{array}$ & $\begin{array}{l}\text { Maximum } \\
\text { Minimum } \\
\text { Mittelwert }\end{array}$ & $\begin{array}{l}24,1 \\
18,5 \\
22,1\end{array}$ & $\begin{array}{l}27,2 \\
16,9 \\
22,7\end{array}$ & $\begin{array}{l}45,4 \\
17,3 \\
28,8\end{array}$ & $\begin{array}{l}40,0 \\
26,5 \\
33,6\end{array}$ & $\begin{array}{l}5,9 \\
0 \\
2,9\end{array}$ & $\begin{array}{l}36,9 \\
19,2 \\
26,0\end{array}$ & $\begin{array}{r}18,2 \\
8,3 \\
13,3\end{array}$ & $\begin{array}{c}10,5 \\
0 \\
4,1\end{array}$ & $\begin{array}{l}26,0 \\
15,0 \\
20,5\end{array}$ \\
\hline $\begin{array}{l}\text { Entstehungs- } \\
\text { zeit der } \mathrm{B}_{1}- \\
\text { Avitaminose } \\
\text { (Tag) }\end{array}$ & $\begin{array}{l}\text { Maximum } \\
\text { Minimum } \\
\text { Mittelwert }\end{array}$ & $X$ & $\begin{array}{l}37 \\
34 \\
38,3\end{array}$ & $\mathbf{X}$ & $\begin{array}{l}70 \\
49 \\
57,0\end{array}$ & $\mathrm{X}$ & $\begin{array}{l}57 \\
33 \\
47,2\end{array}$ & $\mathbf{X}$ & $X$ & $\mathbf{X}$ \\
\hline $\begin{array}{c}\text { Schädigungs- } \\
\text { erscheinung } \\
\text { übermässi- } \\
\text { gen Fetts } \\
\text { (Tag) }\end{array}$ & $\begin{array}{l}\text { Maximum } \\
\text { Minimum } \\
\text { Mittelwert }\end{array}$ & $\mathbf{K}$ & $\mathbf{K}$ & $\mathbf{K}$ & K & $\mathrm{K}$ & $\mathbf{K}$ & $\mathbf{K}$ & $\mathbf{K}$ & $\bar{K}$ \\
\hline $\begin{array}{l}\text { Lebensdauer } \\
\text { (Tag) }\end{array}$ & $\begin{array}{l}\text { Maximum } \\
\text { Minimum } \\
\text { Mittelwert }\end{array}$ & $\begin{array}{c}\$(3) \\
101 \\
102,5 \\
\$(3)\end{array}$ & $\begin{array}{l}53 \\
41 \\
46,3\end{array}$ & $\begin{array}{c}\$(2) \\
75 \\
92,3 \\
\$(2)\end{array}$ & $\begin{array}{l}83 \\
54 \\
63,6\end{array}$ & $\begin{array}{l}\S \\
\S\end{array}$ & $\begin{array}{l}66 \\
38 \\
52\end{array}$ & $\begin{array}{l}\$(2) \\
82 \\
96,0 \\
\$(2)\end{array}$ & $\S$ & $\begin{array}{l}82 \\
70 \\
76\end{array}$ \\
\hline
\end{tabular}

Anm. X...... Keine $B_{1}$-Avitaminose.

K......Keine Schädigungserscheinung über'mässigen Fetts beobachtet.

R.E.......Bedeutet Ratteneinheit.

\section{(a) Mit Butter gefütterte Gruppen.}

1. Zu- und Abnahme des Körpergewichts.

Unter den mit Butter gefäterten Gruppen fällt auf, dass zwischen der mit 50\% und der mit 10\% gefütterten Gruppe kaum ein Unterschied in Körpergewicht zu finden und der Einfluss des Hefezu- 
III.

mit Butter und Lebertran.

\begin{tabular}{|c|c|c|c|c|c|c|c|c|c|c|c|c|}
\hline$x$ & XI & XII & XIII & XIV & $X V$ & XVI & XVII & XVIII & $X I X$ & $\mathrm{xX}$ & $\mathrm{XXI}$ & XXII \\
\hline $\begin{array}{r}100- \\
140\end{array}$ & $\begin{array}{r}150- \\
190\end{array}$ & $200>$ & $\begin{array}{r}100- \\
140\end{array}$ & $\begin{array}{r}1 \text { กิ- } \\
190\end{array}$ & $200>$ & $\begin{array}{r}100- \\
140\end{array}$ & $\begin{array}{r}150- \\
190\end{array}$ & $200>$ & $\begin{array}{r}150- \\
190\end{array}$ & $200>$ & $\begin{array}{r}150- \\
190\end{array}$ & $200>$ \\
\hline $\begin{array}{l}34,09 \\
55,17\end{array}$ & \begin{tabular}{|l}
34,09 \\
55,17
\end{tabular} & \begin{tabular}{|l|l}
34,09 \\
55,17
\end{tabular} & $\begin{array}{l}22,87 \\
43,91\end{array}$ & $\begin{array}{l}22,87 \\
4,3,41\end{array}$ & 22,87 & 22,87 & 22,87 & 22,87 & 17,16 & 17,16 & 17,16 & 17,16 \\
\hline 10,74 & 10,74 & 10,74 & 31,49 & 31,49 & 31,49 & 31,49 & 31,49 & 31,49 & 50,16 & 50,16 & 50,16 & 50,16 \\
\hline $\begin{array}{c}183,8 \\
\text { R.E. }\end{array}$ & $\begin{array}{c}183,8 \\
\text { R.E. }\end{array}$ & $\begin{array}{l}183,8 \\
\text { R.E. }\end{array}$ & $\begin{array}{r}551,5 \\
\text { R.E. } \\
0,2\end{array}$ & $\begin{array}{r}551,5 \\
\text { R.E. } \\
0,2\end{array}$ & $\begin{array}{r}551,5 \\
\text { R.E. } \\
0,2\end{array}$ & $\begin{array}{l}551,5 \\
\text { R.E. }\end{array}$ & $\begin{array}{l}551,5 \\
\text { R.E. }\end{array}$ & $\begin{array}{c}551,5 \\
\text { R.E. }\end{array}$ & $\mid \begin{array}{r}919,1 \\
\text { R.E. } \\
0, \underline{2}\end{array}$ & $\begin{array}{r}919,1 \\
\text { R.E. } \\
0,2\end{array}$ & $\begin{array}{c}919,1 \\
\text { R.E. }\end{array}$ & $\begin{array}{c}919,1 \\
\text { R.E. }\end{array}$ \\
\hline 18,1 & 0 & 6,2 & 44,4 & 15,0 & 4,7 & 10,0 & 0 & 0 & 0 & 0 & 0 & 0 \\
\hline 0 & 0 & 2,2 & 9,0 & 0 & 0 & 9,0 & 0 & 0 & 0 & 0 & 0 & 0 \\
\hline 6,6 & 0 & 4,2 & 26,4 & 8,3 & 3,0 & 9,5 & 0 & 0 & 0 & 0 & 0 & 0 \\
\hline 33,3 & 35,3 & 38,0 & 20,0 & 33,8 & 28,5 & 20,0 & 35,3 & 75,0 & 41,5 & 40,0 & 38,9 & 40,0 \\
\hline 9,0 & 27,2 & 22,0 & 0 & 0 & 0 & 0 & 5,9 & 20,0 & 29,2 & 23,0 & 18,8 & $\begin{array}{l}35,0 \\
0\end{array}$ \\
\hline 25,2 & 31,2 & 25,0 & 14,1 & 13,2 & 18,1 & 10,0 & 21,1 & 43,8 & 37,5 & 30,8 & 28,8 & 37,5 \\
\hline & & & $\mathrm{X}(2)$ & $\mathrm{X}(3)$ & $X(1)$ & & & & & & & \\
\hline & & & 81 & $96(1)$ & 88 & & & & & & & \\
\hline $\mathrm{X}$ & $\mathrm{x}$ & $\mathrm{x}$ & $\begin{array}{c}84,5(2) \\
X(2)\end{array}$ & $\begin{array}{r}96(1) \\
\times(3)\end{array}$ & $\begin{array}{l}90(3) \\
\times(1)\end{array}$ & $\mathrm{x}$ & $\mathrm{X}$ & $x$ & $\mathrm{X}$ & $\mathrm{x}$ & $\mathrm{x}$ & $\mathrm{X}$ \\
\hline & & & 70 & 55 & 55 & 49 & $\mathrm{~K}(3)$ & $\mathrm{K}(3)$ & & & 16 & 16 \\
\hline & & & 43 & 50 & 50 & 33 & $36(1)$ & $49(1)$ & & & 10 & 12 \\
\hline $\mathbf{K}$ & $\mathbf{K}$ & K & 59,0 & 51,0 & 52,2 & 41,0 & $\begin{array}{r}36(1) \\
K(3) \\
\end{array}$ & $\begin{array}{l}49(1) \\
\mathrm{K}(3)\end{array}$ & $\mathbf{K}$ & $\mathbf{K}$ & 13,1 & 14,0 \\
\hline 47 & 42 & 41 & $\$(2)$ & $\$(3)$ & $\$(3)$ & 54 & 47 & 57 & 35 & 44 & 35 & 30 \\
\hline 37 & 31 & 35 & 47 & y9 & 75 & 42 & 32 & 29 & 21 & 23 & 32 & 17 \\
\hline 42,7 & 34,3 & 38 & $\begin{array}{l}98 \\
\$(2)\end{array}$ & $\begin{array}{c}104,5 \\
\$(3)\end{array}$ & $\begin{array}{c}103,5 \\
\S(3)\end{array}$ & 48 & 39,4 & 33,5 & 27,7 & 38,6 & 33,5 & 23,1 \\
\hline
\end{tabular}

$\$$.......Die ganze Versuchszeit hindurch am Leben geblieben.

( ).......Zahl des Versuchstiers.

satzes nur ganz gering ist. Bei der mit $50 \%$ Butter und mit Hefe gefütterten Gruppe findet man die grösste Körpergewichtszunahme.

2. Entstehung der $\mathbf{B}_{1}$-Avitaminose.

Bei der mit 30\% Butter gefütterten Gruppe verzögert sich die Entstehung der $\mathrm{B}_{1}$-Avitaminose am meisten im Vergleich mit den 
Gruppen, die kleine oder grosse Mengen bekamen; bei ihr waren dafür etwa ebensoviel Tage wie bei der mit fettarmer Nahrung gefütterten Gruppe nötig. Bei den mit einer kleinen oder grossen Menge Butter gefütterten Gruppen wird die $\mathrm{B}_{1}$-Avitaminose gefördert. Deswegen kann man hier auch keine sparende Wirkung des Fetts auf Vitamin $B_{1}$ nachweisen.

3. Schädigende Wirkung übermässigen Fetts.

Trotz einer so grossen Menge wie 50\% Butter wurde doch keine schädigende Wirkung durch Fett hervorgerufen. Dabei blieb Hefe ganz ohne Einfluss. Das hängt m. E. von der Eigenschaft des Fetts selbst ab, die eben der Butter eigentümlich ist (S. Abb. 2).

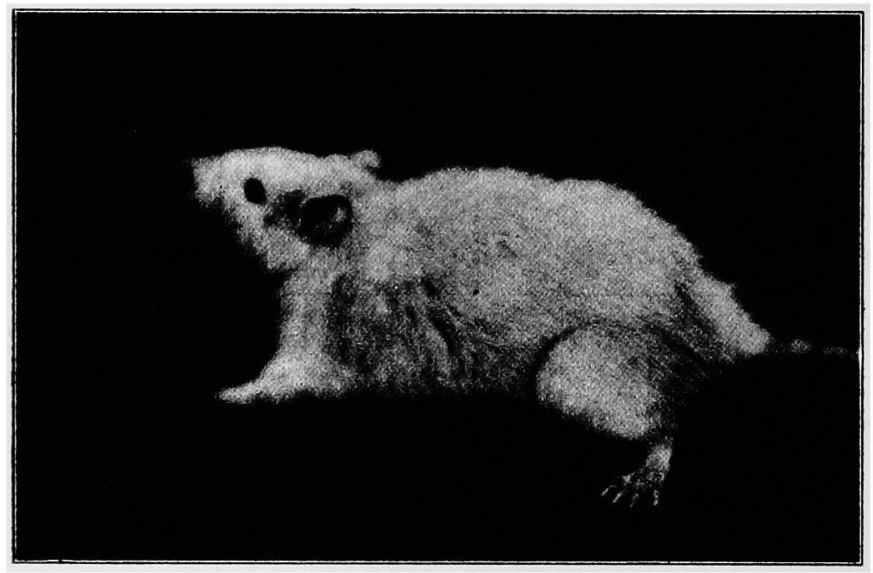

Abb. 2. Das Grundfutter mit 50\% Butter gegeben. (S. Tabelle III, Gruppe VI.) Aufgenommen am 44 Versuchstage.

\section{Lebensdauer.}

Die mit Hefe und Butter gefütterten Gruppen blieben die ganze Versuchszeit hindurch am Leben, während unter den nicht mit Hefe gefütterten Gruppen die mit mittelgrosser Buttermenge gefütterte am längsten lebte. Also ist eine grosse Fettmenge zur Erhaltung des Lebens schädlich; die Hefe kann diesen Schaden beheben.

(b) Mit Lebertran gefüttcrte Gruppen.

1. Zu- und Abnahme des Körpergewichts.

Bei den nicht mit Hefe gefütterten Gruppen wird die Abmage- 
rung desto stärker, je mehr Lebertran genommen wird, und je geringer das Körpergewicht im Anfang, d. h. je jünger das Tier, umso geringere Abmagerung wurde bemerkt. Dabei schwächte Hefegabe die Abmagerung infolge von Lebertranfuitterung ziemlich stark ab, und das Verhältnis zwischen der Menge oder dem Körpergewicht und dem Abmagerungsgrad war ebenso wie bei den nicht mit Hefe gefütterten Gruppen.

\section{Entstehung der $\mathrm{B}_{1}$-Avitaminose.}

Bei allen mit hefefreier Nahrung gefütterten Gruppen tritt ausnahmslos frühzeitiger Tod ein, ehe sich noch $\mathrm{B}_{1}$-A vitaminose einstellt. Unter den mit Hcfe gefütterten Gruppen entsteht keine $\mathrm{B}_{1}$-Avitaminose bei den mit einer kleinen Lebertranmenge gefütterten Tieren; hingegen kann man, ungeachtet des Hefezusatzes, bei den mit einer mittelgrossen Lebertranmenge gefütterten Tieren zumeist Beriberisymptome beobachten. Bei den mit einer grossen Lebertranmenge gefütterten Tieren tritt frühzeitiger Tod ein, aber Beriberisymptome zeigen sich nicht, höchstwahrscheinlich infolge des frühzeitigen Todes. Denn bis zum Entstehen der $\mathrm{B}_{1}$-Avitaminose sind wohl etwa 60 Tage nötig, ganz wie bei der mit fett- oder hefefreier Nahrung gefütterten Gruppe im vorigen Versuch.

Man sieht-also bei übermässiger, d. h. 30-50\%iger Lebertranfütterung das Gegenteil von "sparing action."

3. Schädigende Wirkung übermässigen Fetts.

Unter den nicht mit Hefe gefütterten Gruppen kann man diese Erscheinungen bei denen mit 30\% Lebertran erst unmittelbar vor dem Tod, bei denen mit $50 \%$ Lebertran aber schon sehr früh beobachten.

Vor allem bei der mit 10\% Lebertran gefütterten Gruppe trat der Tod ein, ehe sich die toxische Erscheinungen des Fetts einstellten.

Unter den mit Hefe gefütterten Tieren kann man nur bei denen mit 10\% Lebertranfütterung keine schädigende Wirkung des Fetts beobachten; bei denen mit 30-50\% Lebertranfütterung stimmt die Entstehungszeit der toxischen Erscheinungen im wesentlichen mit der bei den vorigen, mit Baumwollsamenöl gefütterten Gruppen überein.

\section{Lebensdauer.}

Unter den ohne Hefe gefütterten Tieren tritt frühzeitiger Tod im umgekehrten Verhältnis zur Lebertranmenge ein, d. h. je mehr Leber- 
tran, desto früherer Tod. Unter den mit Hefe gefütterten Tieren bleibt die Gruppe mit 10-30\% Lebertran zumeist die ganze Versuchszeit hindurch am Leben; bei Ratten mit grossem Körpergewicht, d. h. bei erwachsenen Ratten kommt der Tod ein wenig früher als bei jungen. Bei den Tieren mit 50\% Lebertran tritt trotz der Hefe der Tod am frühesten ein.

Kurz zusammengefasst: übermässiger Lebertran wirkt Tod fördernd, Hefe dagegen hernmend.

Selbst beim tierischen Fett oder Öl zeigen Lebertran und Butter untereinander verschiedene Erscheinungen, und sogar bei ein und derselben Fettart werden sie von der Menge bestimmt. Im allgemeinen hat die Butter viele dem Lebertran überlegene Eigenschaften. Lebertran in grosser. Menge fördert die toxischen Erscheinungen und zwar umso stärker, je grösser sie ist. Es ist auch bei Butter fraglich, ob es bei ihr eine "sparing action" gibt. Was den Lebertran betrifft, so wird nur bei einer kleinen Menge und Hefezusatz das Leben normal erhalten; bei $30 \%$ oder $50 \%$ Lebertran tritt sehr früh die schädigende Wirkung des übermässigen Fetts ein ; sie fördert deshalb die $B_{1}$-Avitaminose, wenn auch bei Hefegabe in der Regel keine eigentlichen Beriberisymptome auftreten.

Warum haben obige drei Versuchsreihen die Evans-u. Lepkovskyschen Ergebnisse nicht bestätigt? Während ich bei den ersten zwei Versuchsreihen als Öl Baumwollsamenöl verwendet habe, haben Evans u. Lepkovsky 2Tropfen Lebertran genommen und ihn die Rolle des Vitamin A spielen lassen. Dass Vitamin A ein unentbehrlicher Faktor für den Fettstoff wechsel ist, steht schon ganz fest und kommt somit nicht mehr in Frage. Wenn man, wie Evans u. Lepkovsky, als Gegenstand der Forschung die „sparing action of fat on antineuritic Vitamin B" nimmt, so muss man diese Frage m. E. von zwei Seiten aus untersuchen, einmal vom Vitamin A her und dann vom Fett selbst aus. Wie eben erwähnt, haben Evans u. Lepkovsliy pro Tag in das Futter 2Tropfen Lebertran getan, die etwa $0,03 \mathrm{ccm}$ betrugen. Nach Matsuoka $a^{7}$ beträgt 1 Ratteneinheit Vitamin A des Phys.-Chem. Instituts, d. h. Biosterin, 0,01 mg. Wenu man als Standard die Butter mit 100 berechnet, so beträgt Biosterin 3400000 , Lebertran 2000 und somit 1 Ratteneinheit des Biosterins 34, so dass $0,03 \mathrm{ccm}$ Lebertran $75, \boldsymbol{d} . \mathrm{h}$. mehr als 2 Ratteneinheiten, beträgt und daher eine zureichende Menge ist. In dem von Evans u. Lepkovsky verwendeten Baumwollsamenöl und Speck ist nach Fujimaki u. Arimoto $0^{8)}$ und Miura $a^{9)}$ der Vitamin A-Gehalt gleich null oder ganz gering, und nach Schall ${ }^{10}$ kann Speck erst dann die Rolle des Vitamin A spielen, wenn er von der ganzen Nahrung $50 \%$ beträgt. Jedenfalls ist der Vitamin A-Gehalt im Baumwollsamenöl oder Speck sehr gering, ja man kann sagen : fast null. Dass ich bei meiner ersten wie zweiten Versuchsreihe keine mit den Evans- u. Lepkovskyschen Ergebnissen übereinstimmende bekommen habe, dürfte von dem Mangel an 
Vitamin A herrühren. Deshalb habe ich zum Versuch Butter- und Lebertranfütterung benutzt. Doch hat es sich durch diese Versuch herausgestellt, dass diese Fettarten mit reichem Vitamin A keine besondere ,sparing action" entwickeln, ja bei Lebertran sogar auffallend schädlich wirken. Haben Evans u. Lepkorsky auch bei dem mit kohlehydratreicher Nahrung gefütterten Fall hochgradige "sparing action of fat on antineuritic Vitamin B" festgestellt, so habe ich jedenfalls gar nichts Derartiges beobachten können.

Wenn man beim Versuch eine Nahrung verwendet, in der überhaupt mehrere variable Faktoren vorhanden sind, die untereinander in engster Wechselbeziehungen stehen, so muss man die Untersuchung möglichst von allen Seiten aus durchführen. Dass die kompensatorische Wirkung des Fetts bei kohlehy. dratreicher Grundnahrung erheblich ist, beruht nach A b d erhald en ${ }^{11)}$ darauf, dass die Fettfütterung die relative Menge des Kohlehydrats vermindert, dadurch der Kohlehydratstoff wechsel beschräkt wird und folglich der Vitamin $B_{1}$-Bedarf abnimmt. Zum Beweise dafür hat Abderhalden zusammen mit Wertheim e ${ }^{12}$ die Beziehungen zwischen dem Leber- und Herzglykogengehalt und dem Krampfe bei $B_{1}$-Avitaminose untersucht. Danach übt Vitamin $B_{\text {, }}$ einen mächtigen Einfluss auf den Kohlehydratstoff wechsel infolge des bei $B_{1}$. Avitaminose auftretenden Krampfes aus, weil die Krämpfe erst durch Vitamin $\mathbf{B}_{\mathbf{1}}$ beseitigt werden und dabei zugleich das angehäufte Leberglykogen zum normalen Wert zurückkehrt.

Dass der Vitamin B-Komplex die Assimilation sowie die Dissimilation von Kohlehydrat, Eiweiss und Fett in gleicher Weise fördert und reguliert; und einen wichtigen Faktor für die Lebenserhaltung bildet, haben die Forschungen von Asada, ${ }^{13}$ ) Bickel, ${ }^{14)}$ Bickel . Kaufmann,${ }^{15)}$ Tscherkes, ${ }^{16}$ ) Yama$\mathrm{da},{ }^{1}{ }^{\text {i) }} \mathrm{Koll} \mathrm{at}^{18)} \mathrm{u}$. a. erwiesen, und das ist schon allgemein anerkannt.

Wenn einer Nahrung mit einem der drei Elemente im Überfluss zu viel Vitamin B-Komplex fehlt, so entsteht, je mehr Kohlehydrate vorhanden, umso früher $B_{1}$-Avitaminose $\left(F \mathrm{nk}^{19)}\right)$; je mehr Kohlehydrate vorhanden, umso grösser wird der Vitamin B-Komplex-Bedarf (Collazo u. Pi-Suner Ba y ${ }^{20}$ ), insbesondere soll Vitamin $B_{1}$ beim Kohlehydratstoff wechsel eine wichtige Rolle spielen (Abderhalden, Kaufmann, Vasiricou. Oerin ${ }^{21}$ ).

Wenn man im übrigen, wie Evans es getan, auch die Körpergewichtszunahme und die Lebensdauer als Kennzeichen nimmt, so kann man einerseits, wie Inoue,22) beobachten, dass Kohlehydrate sehr günstig auf junge Ratten wirken und mässig stark auf erwachsene Ratten. Anderseits fand Mats noka, ${ }^{23}$ dass bei traubenzuckerreichem Futter die Entwicklung sich umso günstiger vollzieht, je mehr Hefe vorhanden. Hieraus ist eine sehr enge Beziehung $z$ wischen Kohlehydratstoff wechsel und Vitamin B-Komplex oder Vitamin $B_{2}$ ersichtlich.

Hinsichtlich der Beziehung zwischen Eiweiss und $B_{1}$-Avitaminoseentstehung haben zwar Funk $u$. D ubin ${ }^{2+)}$ und Abderhalde ${ }^{25}$ ) Hemmung durch Eiweiss behauptet, aber in letzter Zeit halten nur noch wenige Autoren diese Theorie aufrecht. Osborn u. Mendel, ${ }^{2(6) 28)}$ Addis, MacKay u. MacKay, ${ }^{2 i)}$ Francis, Smith u. Mo is $\mathrm{e}^{29}$ u. a. haben keine quantitativen Beziehungen zwischen den beiden Elementen gefunden. Dagegen wird $B_{1}$-Avitaminose nach 
Tscherkes, ${ }^{16) 30)}$ Reader u. Drummond, ${ }^{31)}$ Hassan u. a., ${ }^{32}$ ) Hartwell, ${ }^{33}$ ) Plimmer, ${ }^{34)}$ Francis, Smith u. Mendel ${ }^{35}$ ) und Prunty u. Roscoe $e^{35}$ ) desto stärker gefördert, je mehr Eiweiss vorhanden ist. Auf Grund dieser neueren Forschungen möchte ich annehmen, dass der Vitamin B-Komplex umso grösser ist, je mehr Kohlehydrate vorhanden sind und dass eine grosse Menge Eiweiss die Entstehung der B-Komplex-Avitaminose fördert. Evans u. Lepkorsky sehen auch in einer kohlehydratreichen Grundnahrung die beste Bedingung für die ,sparing action of fat on antineuritic Vitamin B". Wenn das die Entstehung der B-Komplex-Avitaminose fördernde Eiweiss nur in kleiner Menge vorhanden ist und zugleich viel Fett oder Öl gegeben wird, so muss der Vitamin B-Komplex-Bedarf klein sein, weil das Vitamin B-Bedürfnis wegen der kleinen Menge der benutzten Kohlehydrate sehr gering ist. Eben deshalb könnte die Abderhaldensche Theorie eher recht haben als die "sparing action"-Theorie.

Will man aber ammehmen, dass irgendeine ,sparing action of fat on antineuritic Vitamin B" wirksam ist, so bleibt noch die Frage offen, ob das Fett selbst oder das ihm anhaftende Vitamin A diese Wirkung ausübt.

Das thermostabile Vitamin B hat wohl bei den Versuchen an Tauben die Schädigung durch übermässiges Fett und die Verklebung des mit öl getränkten Gefieders gehemmt, bei den Versuchen an Ratten aber hat es gar keine Wirksamkeit gezeigt. Woher kommt das? Nach Williams u. Waterman $n^{37)^{38}}$ ist Vitamin $B_{3}$, das thermolabiler ist, der Hauptfaktor zur Körpergewichtserhaltung und Entwicklungsförderung bei Tauben. Hier kommt nicht dieser Faktor, sonderu vielmehr der thermostabile Peter ssche ${ }^{39}$ ) Faktor in Betracht, der dieselbe biologische Bedeutung wie Vitamin $B_{3}$ hat und von $P$ eters Vitamin $B_{5}$ genannt wurde. Diesen Faktor háben Reader, Carter $\mathbf{u}$. Kinnesles $y^{(0)}$ in ihren Nachprüfungen ermittelt und ihre Ergebnisse darüber veröffentlicht. Das thermostabile Vitamin $B_{2}$ könnte nach György, Kuhnu. Wagner-J a u re g g $^{+1}$ ) eine Art Flavinstoff sein; es wirkt niemals unabhängig, und zu seiner Wirkung ist irgendein Hilfsmaterial nötig. Bei Ratten soll der Faktor zur Lebenserhaltung und Entwicklungsförderung Vitamin $\mathrm{B}_{4}$ sein, das thermolabil ist (Reader $;^{42+13)}$ Halliday, $\left.\mathrm{Nunn} \mathrm{u.} \mathrm{Fischer}{ }^{44}\right)$ ).

Nach Obigem ist Vitamin B ein Komplex verschiedener Faktoren, unter denen wir den thermolabilen und den thermostabilen unterscheiden kömnen. Auch nach meinen Versuchen dürfte man annehmen, dass der bisher Vitamin $B_{2}$ genannte thermostabile Faktor ebenfalls ein Komplex ist und niemals unabhängig wirkt. Also liesse es sich unschwer denken, dass bei Tauben eben darum die bei Ratten nicht nachgewiesene Verzögerung der schädigenden Wirkung übermässigen Fetts hervorgernfen wurde, weil bei Tauben das Gy ö rgysche Vitamin $B_{2}$ und das Peterssche Vitamin $B_{5}$ nebeneinander vorhanden waren und miteinander synergetisch gewirkt haben.

\section{Zusammenfassung des ersten Teils.}

1. Evans' u. Lepkovskys, ,sparing action of fat on antineuritic Vitamin $B^{\prime \prime}$ ist weder beim vegetabilischen Öl d. h. bei Baum- 
wollsamenöl ohne Vitamin A, noch beim animalischen Fett und öl, d. h. bei Butter und Lebertran mit Vitamin A, festzustellen.

2. Bei Fett oder Öl ist gar keine sparende Wirkung auf Vita$\min \mathrm{B}_{1}$ nachweisbar.

3. Bei Baumwollsamenöl ohne Vitamin A wirkt übermässiges Fett umso früher schädigend, je mehr davon gegeben wird, und somit scheint die $\mathrm{B}_{1}$-Avitaminose dadurch gefördert zu werden. Auch bei Lebertran, mit einer grossen Menge Vitamin A, werden selbst im Fall einer mittelgrossen Menge, zusammen mit Hefe, bei dem überhaupt keine $\mathrm{B}_{1}$-Avitaminose einzutreten pflegt, Beriberi-Symptome hervorgerufen.

Die Butter hat die besondere Eigenschaft, dass sie weder fördernd noch verzögernd wirkt, wieviel man auch davon geben mag.

4. Durch Baumwollsamenöl und Lebertran werden Körpergewichtsabnahme, Abmagerung und frühzeitiger Tod hervorgerufen, aber bei Butter ist dies selbst bei einer grossen Menge nicht der Fall. Die Vitamin B-Faktoren üben eine antagonistische oder synergetische Wirkung auf die Schädigung durch übermässiges Fett aus.

\section{Zweiter Teil.}

Versuche mit verschiedenen Zusammensetzungen einiger Fettund Ölarten, Vitamin A- und Vitamin B-Faktoren.

Um die in den vorigen Versuchen behandelte, aber noch nicht erledigte Frage nach der Wechselbeziehung z wischen Fett oder Öl, Vitamin, A, Vitamin B-Komplex und thermostabilem Vitamin B zu klären, habe ich die folgenden Versuche angestellt. Als Fett wurden Baumwollsamenöl, Speck, Butter und Lebertran verwandt, als Vitamin A Biosterin (Phys.-Chem. Inst.), als Vitamin B-Komplex Hefe und als thermostabiler Vitamin B-Faktor die im Autoklav erhitzte Hefe.

Die Zusammensetzung der drei Elemente der Nahrung war immer dieselbe (d. h. Eiweiss...27,0\%, Kohlehydrate...49,7\% und Fett ...23,2\%); Vitamin wurden in verschiedener Menge hinzugefügt. Jede Gruppe bestand aus 4 Ratten. Die Versuchsergebnisse sind in der folgenden Tabelle zusammengestellt.

Als fettfreie Nahrung habe ich ein 24 Stunden lang in Alkohol, dann 24 Stunden lang in Äther extrahiertes und danach getrocknetes Gemisch von Gasëin, Reismehl und Zucker verwandt. Die Hefe habe ich für die fettfreie Nahrung nicht entfettet, weil die Menge der Hefe sehr klein ist; der Fettgehalt in $0,2 \mathrm{~g}$ Hefe beträgt nur 4,8 mg. 
Versuchsergebnisse.

1. Zu- und Abnahme des Körpergewichts.

(a) Versuche ohne Vitamin A.

Auf das Körpergewicht der Gruppe ohne Vitamin B-Faktoren übt

Tabelle

Versuche mit verschiedenen Zusammensetzungen einiger

\begin{tabular}{|c|c|c|c|c|c|c|c|}
\hline \multicolumn{2}{|c|}{ Gruppe } & I & II & III & IV & $\mathrm{V}$ & VI \\
\hline $\begin{array}{c}\text { Bestandteile } \\
\text { des Futter's } \\
(\%)\end{array}$ & $\begin{array}{l}\text { Eiweiss } \\
\text { Kohlehydrat } \\
\text { Fett }\left\{\begin{array}{l}\text { Butter } \\
\text { Lebertran } \\
\text { Baumwoll- } \\
\text { samenöl } \\
\text { Speck }\end{array}\right. \\
\end{array}$ & $\begin{array}{l}35,08 \\
64,39\end{array}$ & $\begin{array}{l}35,08 \\
64,39\end{array}$ & $\begin{array}{l}35,08 \\
64,39\end{array}$ & $\begin{array}{l}27,07 \\
49,68 \\
23,23\end{array}$ & $\begin{array}{l}27,07 \\
49,68 \\
\\
23,23\end{array}$ & $\begin{array}{l}27,07 \\
49,68 \\
23,23\end{array}$ \\
\hline Vitamin & 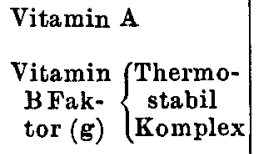 & $\begin{array}{r}1 / 2 \\
\text { R.E. }\end{array}$ & $\begin{array}{l}1 / 2 \\
0,2\end{array}$ & 0,2 & $\begin{array}{l}11,8 \\
\text { R.E. }\end{array}$ & $\begin{array}{r}11,8 \\
0,2\end{array}$ & $\begin{array}{r}11,8 \\
0,2\end{array}$ \\
\hline $\begin{array}{l}\text { Körperge- } \\
\text { wichtszu- } \\
\text { nahme } \\
(\%)\end{array}$ & $\begin{array}{l}\text { Maximum } \\
\text { Minimum } \\
\text { Mittelwert }\end{array}$ & $\begin{array}{r}25,0 \\
8,8 \\
16,8\end{array}$ & $\begin{array}{c}16,0 \\
0 \\
9,5\end{array}$ & $\begin{array}{l}20,0 \\
10,0 \\
15,0\end{array}$ & $\begin{array}{l}16,7 \\
11,1 \\
12,8\end{array}$ & $\begin{array}{l}38,9 \\
11,1 \\
23,9\end{array}$ & $\begin{array}{r}60,0 \\
9,0 \\
30,7\end{array}$ \\
\hline $\begin{array}{l}\text { Körperge- } \\
\text { wichtsab- } \\
\text { nahme } \\
(\%)\end{array}$ & $\begin{array}{l}\text { Maximum } \\
\text { Minimum } \\
\text { Mittelwert }\end{array}$ & $\begin{array}{c}12,5 \\
0 \\
3,1\end{array}$ & $\begin{array}{l}42,0 \\
17,0 \\
30,5\end{array}$ & $\begin{array}{l}0 \\
0 \\
0\end{array}$ & $\begin{array}{l}27,8 \\
11,1 \\
16,9\end{array}$ & $\begin{array}{l}38,9 \\
11,1 \\
12,6\end{array}$ & $\begin{array}{l}0 \\
0 \\
0\end{array}$ \\
\hline $\begin{array}{c}\text { Entstehungs- } \\
\text { zeit der } \mathbf{B}_{1}- \\
\text { Avitaminose } \\
\text { (Tag) }\end{array}$ & $\begin{array}{l}\text { Maximum } \\
\text { Minimum } \\
\text { Mittelwert }\end{array}$ & $\mathbf{x}$ & $\begin{array}{l}55 \\
47 \\
53\end{array}$ & $\begin{array}{l}47 \\
28 \\
37,5\end{array}$ & $\begin{array}{l}X(1) \\
50 \\
56(3) \\
X(1) \\
\end{array}$ & $\begin{array}{l}90 \\
84 \\
87\end{array}$ & $\mathbf{X}$ \\
\hline $\begin{array}{c}\text { Schädigungser- } \\
\text { scheinung über- } \\
\text { mässigen Fetts } \\
\text { (Tag) }\end{array}$ & $\begin{array}{l}\text { Maximum } \\
\text { Minimum } \\
\text { Mittelwert }\end{array}$ & $\mathbf{K}$ & $\mathbf{K}$ & $\mathbf{K}$ & $\mathbf{K}$ & $\mathbf{K}$ & $\mathbf{K}$ \\
\hline $\begin{array}{l}\text { Lebensdauer } \\
\text { (Tag) }\end{array}$ & $\begin{array}{l}\text { Maximum } \\
\text { Minimum } \\
\text { Mittelwert }\end{array}$ & $\begin{array}{l}61 \\
37 \\
47,0\end{array}$ & $\begin{array}{l}72 \\
57 \\
65,7\end{array}$ & $\begin{array}{l}63 \\
47 \\
55,0\end{array}$ & $\begin{array}{l}74 \\
52 \\
59,5\end{array}$ & $\begin{array}{l}77 \\
85 \\
92,0\end{array}$ & $\S$ \\
\hline
\end{tabular}

Anm. \$...... Die ganze Versuchszeit hindurch am Leben geblieben.

( ).......Zahl des Versuchstiers.

$\mathrm{X}$........ Keine $\mathrm{B}_{1}$-A vitaminose. 
Speck oder Baumwollsamenöl einen sehr schlechten Einfluss aus, d. h. das Körpergewicht nahm dadurch erheblich ab.

Bei den Gruppen mit thermostabilem Vitamin B wirkt Speckoder Baumwollsamenölzusatz auch nicht günstig auf das Körpergewicht.

IV.

Fett- und Ölarten, Vitamin A und Vitamin B-Faktoren.

\begin{tabular}{|c|c|c|c|c|c|c|c|c|c|c|}
\hline VII & VIII & IX & $\mathrm{x}$ & $X I$ & XII & XIII & XIV & $\mathrm{XV}$ & XVI & XVII \\
\hline $\begin{array}{l}27,07 \\
49,68\end{array}$ & $\begin{array}{l}27,07 \\
49,68\end{array}$ & $\begin{array}{l}27,07 \\
49,68\end{array}$ & $\begin{array}{l}27,07 \\
49,68\end{array}$ & $\begin{array}{l}27,07 \\
49,68\end{array}$ & $\begin{array}{l}27,07 \\
49,68\end{array}$ & $\begin{array}{l}27,07 \\
49,68\end{array}$ & $\begin{array}{l}27,07 \\
49,68\end{array}$ & $\begin{array}{l}27,07 \\
49,68\end{array}$ & $\begin{array}{l}27,07 \\
49,68\end{array}$ & $\begin{array}{l}27,07 \\
49,68\end{array}$ \\
\hline 23,23 & 23,23 & 23,23 & 23,23 & 23,23 & 23,23 & 23,23 & 23,23 & 23,23 & 23,23 & 23,23 \\
\hline $\begin{array}{l}29,4 \\
\text { R.E. }\end{array}$ & $\begin{array}{c}29,4 \\
\text { R.E. } \\
0,2\end{array}$ & $\begin{array}{r}29,4 \\
\text { R.E. } \\
\quad 0,2\end{array}$ & 0 & $\begin{array}{l}0 \\
0,2\end{array}$ & 0,2 & $\stackrel{1}{\text { R.E. }}$ & $\begin{array}{c}1 \\
\text { R.E. } \\
0,2\end{array}$ & $\begin{array}{c}1 \\
\text { R.E. } \\
0,2\end{array}$ & $\begin{array}{l}50 \\
\text { R.E. }\end{array}$ & $\begin{array}{l}50 \\
\text { R.E. } \\
0,2\end{array}$ \\
\hline $\begin{array}{r}11,8 \\
5,5 \\
7,2\end{array}$ & $\begin{array}{l}\mathbf{5 , 3} \\
0 \\
\mathbf{3 , 7}\end{array}$ & $\begin{array}{c}50,0 \\
0 \\
25,7\end{array}$ & $\begin{array}{r}23,5 \\
5,9 \\
12,8\end{array}$ & $\begin{array}{r}21,4 \\
8,0 \\
16,7\end{array}$ & $\begin{array}{l}50,0 \\
18,8 \\
31,9\end{array}$ & $\begin{array}{c}10,5 \\
0 \\
3,8\end{array}$ & $\begin{array}{c}11,8 \\
0 \\
4,3\end{array}$ & $\begin{array}{r}26,6 \\
6,3 \\
18,1\end{array}$ & $\begin{array}{c}12,5 \\
0 \\
5,9\end{array}$ & $\begin{array}{c}11,7 \\
0 \\
8,6\end{array}$ \\
\hline $\begin{array}{l}38,9 \\
17,7 \\
27,8\end{array}$ & $\begin{array}{r}40,0 \\
4,6 \\
17,7\end{array}$ & $\begin{array}{c}35,3 \\
0 \\
15,8\end{array}$ & $\begin{array}{l}40,0 \\
10,0 \\
31,8\end{array}$ & $\begin{array}{r}28,0 \\
7,1 \\
18,2\end{array}$ & $\begin{array}{r}18,8 \\
5,0 \\
5,9\end{array}$ & $\begin{array}{l}33,3 \\
21,1 \\
30,3\end{array}$ & $\begin{array}{l}37,4 \\
18,7 \\
26,1\end{array}$ & $\begin{array}{l}31,3 \\
13,3 \\
21,0\end{array}$ & $\begin{array}{l}29,4 \\
25,0 \\
26,3\end{array}$ & $\begin{array}{c}22,0 \\
0 \\
8,5\end{array}$ \\
\hline 47 & $\mathbf{X}(\mathbf{2})$ & & 47 & 70 & $\mathbf{X}(1)$ & 63 & 75 & & 65 & 57 \\
\hline 39 & $57(2)$ & & 47 & 42 & 80 & 57 & 55 & & 53 & 63 \\
\hline 44,6 & $\begin{array}{l}57(2) \\
\times(2) \\
\end{array}$ & $\mathbf{X}$ & 47 & 51,5 & $\begin{array}{l}83(2) \\
X(1) \\
\end{array}$ & 60,3 & 66,5 & $\mathbf{X}$ & 58,5 & 57,3 \\
\hline $\begin{array}{l}47 \\
37 \\
43,5\end{array}$ & $\begin{array}{l}47 \\
25 \\
34,2\end{array}$ & $\begin{array}{c}K(1) \\
46 \\
55,6(3)\end{array}$ & $\begin{array}{l}47 \\
47 \\
47\end{array}$ & $\begin{array}{l}70 \\
42 \\
51,5\end{array}$ & $\begin{array}{l}55 \\
55 \\
55\end{array}$ & $\begin{array}{l}28 \\
28 \\
28\end{array}$ & $\begin{array}{l}73 \\
24 \\
58,7\end{array}$ & $\begin{array}{l}84 \\
84 \\
84\end{array}$ & $\begin{array}{l}50 \\
24 \\
42\end{array}$ & $\begin{array}{l}57 \\
55 \\
55,6\end{array}$ \\
\hline 68 & 84 & $\$(1)$ & 62 & 85 & $\$(2)$ & 72 & 89 & $\S(3)$ & 72 & 69 \\
\hline 48 & 40 & 69 & 53 & 48 & $97(2)$ & 62 & 71 & $89(1)$ & 66 & 58 \\
\hline 58 & 60,5 & $\begin{array}{c}81.3(3) \\
8(1)\end{array}$ & 59,8 & 69,3 & $\begin{array}{c}97(2) \\
\$(2)\end{array}$ & 65,7 & 77,0 & $\begin{array}{l}89(1) \\
\S(3)\end{array}$ & 69 & 63,5 \\
\hline
\end{tabular}

K...... Keine Schädigungserscheinung übermässigen Fetts beobachtet.

R.E....... Bedentet Ratteneinheit. 


\begin{tabular}{|c|c|c|c|c|c|c|c|}
\hline \multicolumn{2}{|c|}{ Gruppe } & XVIII & XIX & $\mathrm{Xx}$ & XXI & XXII & XXIII \\
\hline $\begin{array}{c}\text { Bestandteile } \\
\text { des Futters } \\
(\%)\end{array}$ & $\begin{array}{l}\text { Eiweiss } \\
\text { Kohlehydrat } \\
\text { Fett }\left\{\begin{array}{l}\text { Butter } \\
\text { Lebertran } \\
\text { Baumwoll- } \\
\text { samenöl } \\
\text { Speck }\end{array}\right.\end{array}$ & $\begin{array}{l}27,07 \\
49,68 \\
\\
23,23\end{array}$ & $\begin{array}{l}27,07 \\
49,68 \\
23,23\end{array}$ & $\begin{array}{r}27,07 \\
49,68 \\
\\
23,23\end{array}$ & $\begin{array}{r}27,07 \\
49,68 \\
23,23\end{array}$ & $\begin{array}{l}27,07 \\
49,68\end{array}$ & $\begin{array}{r}27,07 \\
49,68\end{array}$ \\
\hline Vitamin & 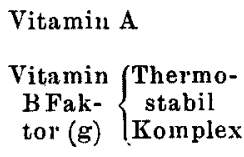 & $\begin{array}{l}50 \\
\text { R.E. } \\
\\
0,2\end{array}$ & $\begin{array}{l}200 \\
\text { R.E. }\end{array}$ & $\begin{array}{l}200 \\
\text { R.E. } \\
0,2\end{array}$ & $\begin{array}{l}200 \\
\text { R.E. } \\
\\
0,2\end{array}$ & 0 & $\begin{array}{l}0 \\
0,2\end{array}$ \\
\hline $\begin{array}{c}\text { Körperge- } \\
\text { wichtszu- } \\
\text { nahme } \\
(\%)\end{array}$ & $\begin{array}{l}\text { Maximum } \\
\text { Minimum } \\
\text { Mittelwelt }\end{array}$ & $\begin{array}{c}42,8 \\
0 \\
14,3\end{array}$ & $\begin{array}{l}6.3 \\
0 \\
2,9\end{array}$ & $\begin{array}{r}30.0 \\
4,7 \\
16,7\end{array}$ & $\begin{array}{c}23,8 \\
0 \\
13,1\end{array}$ & $\begin{array}{r}20,0 \\
5,9 \\
14,2\end{array}$ & $\begin{array}{r}28,6 \\
6,2 \\
13,6\end{array}$ \\
\hline $\begin{array}{c}\text { Körperge- } \\
\text { wichtsab- } \\
\text { nahme } \\
(\%)\end{array}$ & $\begin{array}{l}\text { Maximum } \\
\text { Minimum } \\
\text { Mittelwert }\end{array}$ & $\begin{array}{c}37,5 \\
0 \\
19,9\end{array}$ & $\begin{array}{r}41,0 \\
5,5 \\
17,4\end{array}$ & $\begin{array}{c}23,8 \\
0 \\
12,9\end{array}$ & $\begin{array}{r}38,9 \\
4,7 \\
11,9\end{array}$ & $\begin{array}{l}41,2 \\
20,0 \\
22,7\end{array}$ & $\begin{array}{l}33,3 \\
13,2 \\
27,3\end{array}$ \\
\hline $\begin{array}{l}\text { Entstehungs- } \\
\text { zeit der } \mathrm{B}_{1}^{-} \\
\text {Avitaminose } \\
\text { (Tag) }\end{array}$ & $\begin{array}{l}\text { Maximum } \\
\text { Minimum } \\
\text { Mittelwert }\end{array}$ & $\mathbf{X}$ & $\begin{array}{l}50 \\
29 \\
39,8\end{array}$ & $\begin{array}{l}\mathrm{X}(2) \\
72 \\
79(2) \\
\mathrm{X}(2) \\
\end{array}$ & $\begin{array}{l}\mathrm{X}(2) \\
87 \\
89(2) \\
\mathrm{X}(2) \\
\end{array}$ & $\begin{array}{l}62 \\
60 \\
61\end{array}$ & $\begin{array}{l}62 \\
57 \\
59,2\end{array}$ \\
\hline $\begin{array}{c}\text { Schädigungser- } \\
\text { scheinung über- } \\
\text { mässigen Fetts } \\
\text { (Tag) }\end{array}$ & $\begin{array}{l}\text { Maximum } \\
\text { Minimum } \\
\text { Mittelwert }\end{array}$ & $\begin{array}{l}84 \\
66 \\
76\end{array}$ & $\begin{array}{l}47 \\
23 \\
36\end{array}$ & $\begin{array}{l}65 \\
55 \\
59,3\end{array}$ & $\begin{array}{l}49 \\
37 \\
46\end{array}$ & $\mathbf{K}$ & $\mathbf{K}$ \\
\hline $\begin{array}{l}\text { Lebensdaner } \\
\text { (Tag) }\end{array}$ & $\begin{array}{l}\text { Maximum } \\
\text { Minimum } \\
\text { Mittelwert }\end{array}$ & $\begin{array}{c}\S(3) \\
92(1) \\
92(1) \\
\S(3)\end{array}$ & $\begin{array}{l}61 \\
34 \\
51,2\end{array}$ & $\begin{array}{l}91 \\
80 \\
85\end{array}$ & $\begin{array}{l}\$(2) \\
94(2) \\
94(2) \\
\$(2)\end{array}$ & $\begin{array}{l}66 \\
43 \\
57,3\end{array}$ & $\begin{array}{l}82 \\
71 \\
75,0\end{array}$ \\
\hline
\end{tabular}

Bei den Tieren mit dem Vitamin B-Komplex beobachtete man eine günstigere Wirkung des Fetts oder Öls als bei den vorigen zwei Gruppen.

(b) Versuche mit Vitamin A.

Bei den Ratten ohne Vitamin B-Faktoren schädigt Fett- oder Ölzusatz die Körpergewichtserhaltung stärker als bei denen ohne Fettoder Ölfütterung.

Die Menge des Vitamin A übt hier keinen besonderen Einfluss aus. Auch unter den Gruppen mit Vitamin A und thermostabilem 


\begin{tabular}{|c|c|c|c|c|c|c|c|c|c|}
\hline XXIV & $\mathrm{XxV}$ & XXVI & XXVII & XXVIII & XXIX & $\mathrm{xxx}$ & XXXI & XXXII & XXXIII \\
\hline $\begin{array}{l}27,07 \\
49,68\end{array}$ & $\begin{array}{l}27,07 \\
49,68\end{array}$ & 27,07 & 27,07 & 27,07 & 27,07 & 27,07 & 27,07 & 27,07 & 27,07 \\
\hline & 49,68 & 49,68 & 49,68 & 49,68 & 49,68 & 49,68 & 49,68 & 49,68 & 49,68 \\
\hline 23,23 & 23,23 & 23,23 & 23,23 & 23,23 & 23,23 & 23,23 & 23,23 & 23,23 & 23,23 \\
\hline 0 & $\begin{array}{c}1 \\
\text { R.E. }\end{array}$ & $\begin{array}{c}1 \\
\text { R.E. } \\
0,2\end{array}$ & $\begin{array}{c}1 \\
\text { R.E. }\end{array}$ & $\begin{array}{c}50 \\
\text { R.E. }\end{array}$ & $\begin{array}{c}50 \\
\text { R.E. } \\
0,2\end{array}$ & $\begin{array}{l}50 \\
\text { R.E. }\end{array}$ & $\begin{array}{l}200 \\
\text { R.E. }\end{array}$ & $\begin{array}{c}200 \\
\text { R E. } \\
0,2\end{array}$ & $\begin{array}{l}200 \\
\text { R.E. }\end{array}$ \\
\hline 0,2 & & & 0,2 & & & 0,2 & & & 0,2 \\
\hline $\begin{array}{r}15,0 \\
5,6\end{array}$ & $\begin{array}{c}21,1 \\
0\end{array}$ & $\begin{array}{c}55,5 \\
0\end{array}$ & $\begin{array}{l}14,2 \\
11,1\end{array}$ & $\begin{array}{r}21,5 \\
5,0\end{array}$ & $\begin{array}{l}38,9 \\
11,1\end{array}$ & $\begin{array}{r}19,0 \\
5,9\end{array}$ & $\begin{array}{r}28,6 \\
7,2\end{array}$ & $\begin{array}{l}23,8 \\
14,2\end{array}$ & $\begin{array}{c}31,3 \\
0\end{array}$ \\
\hline 10,2 & 14,8 & 21,0 & 12,9 & 12,8 & 25,8 & $\begin{array}{r}0,8 \\
13,2\end{array}$ & $\begin{array}{r}18,4 \\
18,6\end{array}$ & $\begin{array}{l}14,6 \\
19,3\end{array}$ & $\begin{array}{c}0 \\
15,9\end{array}$ \\
\hline 30,0 & 40,0 & 28,6 & 28,6 & 29,4 & 33,3 & 5,9 & 35,7 & 23,8 & 27,7 \\
\hline 6,3 & 10,5 & 7,6 & 6,2 & 25,0 & 0 & 4,3 & 5,0 & 19,0 & 6,3 \\
\hline 14,7 & $27, \tilde{5}$ & 11,9 & 20,0 & 28,1 & 14,6 & 5,0 & 24,6 & 21,8 & 12,8 \\
\hline & $\mathrm{X}(2)$ & $x(2)$ & & $\mathrm{X}(\mathbf{2})$ & $x(3)$ & & 58 & 75 & \\
\hline & 69 & 69 & & 56 & 75 & & 58 & 75 & \\
\hline $\mathrm{x}$ & $\begin{array}{l}74(2) \\
\times(2) \\
\end{array}$ & $\begin{array}{l}70(2) \\
\mathrm{X}(2) \\
\end{array}$ & $\mathrm{x}$ & $\begin{array}{c}66,5(2) \\
\times(2)\end{array}$ & $\begin{array}{l}75(1) \\
\times(3)\end{array}$ & $\mathrm{x}$ & 58 & 75 & $\mathrm{x}$ \\
\hline $\mathbf{K}$ & $\begin{array}{c}\mathrm{K}(1) \\
55 \\
57,3(3) \\
\mathbf{K}(1) \\
\end{array}$ & $\begin{array}{c}\mathrm{K}(1) \\
53 \\
66,6(3) \\
\mathrm{K}(1) \\
\end{array}$ & K & $\begin{array}{l}\mathrm{K}(3) \\
63 \\
63(1)\end{array}$ & $\begin{array}{l}\mathrm{K}(2) \\
75 \\
75(2)\end{array}$ & $\mathbf{K}$ & $\mathbf{K}$ & $\mathbf{K}$ & $\mathbf{K}$ \\
\hline$\S$ & 88 & 77 & $\S$ & 76 & 82 & $\S(3)$ & 70 & 104 & $\S$ \\
\hline & 54 & 53 & & 69 & 77 & $108(1)$ & 60 & 89 & \\
\hline$\S$ & 73,0 & 68,0 & $\S$ & 70,5 & 80,3 & $\begin{array}{c}108(1) \\
\S(3)\end{array}$ & 63,3 & 93,0 & $\S$ \\
\hline
\end{tabular}

Vitamin B kann man bei den Tieren mit Butter und Speck weniger schädigende Wirkung beobachten, während die Abnahme bei denen mit Baumwollsamenöl oder Lebertran, im Vergleich zu denen ohne Vitamin B-Faktoren und zu denen mit fettfreier Nahrung, überhaupt leichten Grades ist.

Bei den mit Fett gefütterten Gruppen mit dem Vitamin B-Komplex und Vitamin A ist die Zunahme übereinstimmend hohen Grades, bei den Gruppen mit Baumwollsamenöl oder Lebertran sogar auffallend. Auch die Abnahme ist im allgemeinen gering. Denn der 
Vitamin B-Komplex, zusammen mit Fett oder öl und Vitamin A, fördert die Zunahme und hemmt zugleich die Abnahme. Da die Menge des Vitamin A keine Beziehung zur Körpergewichtserhaltung hat, so dürfte sich die Störung der Körpergewichtserhaltung auf die Toxizität des Fetts selbst zurückführen lassen, und die Vitamin B-Faktoren beseitigen diese, denn sie regulieren den Stoffwechsel.

\section{Entstehung der $\mathrm{B}_{1}$-Avitaminose.}

(a) Versuche ohne Vitamin A.

Bei den Tieren mit Speck oder Baumwollsamenöl ist keine ,sparing action" nachweisbar, bei der Gruppe mit Baumwollsamenöl zeigt sich sogar umgekehrt eine die $B_{1}$-A vitaminose fördernde Wirkung. Zusatz von thermostabilem Vitamin B übt keine besondere Wirkung aus. Trotz dem Vitamin B-Komplex wurden bei der Hälfte der' Ratten in der Gruppe mit Baumwollsamenöl Beriberisymptome hervorgerufen. All dies könnte durch die Toxizität des Fetts oder Öls selbst verursacht werden.

(b) Versuche mit Vitamin A.

Bei den Gruppen ohne Vitamin B-Faktoren erscheint die die $\mathrm{B}_{1}$ Avitaminose fördernde Wirkung von Speck und Baumwollsamenöl umso auffallender, je mehr Vitamin A vorhanden ist; nur dass bei der Gruppe mit Speck und einer kleinen Menge Vitamin A die Entstehung der $B_{1}$-Avitaminose ein wenig verzögert wird. Hier scheint die $B_{1}-$ Avitaminose stärker gefördert zu sein als bei den vorigen Fällen ohne Vitamin A.

Nach Obigem scheint Vitamin A die $B_{1}$-Avitaminose zu fördern. Unter den Gruppen mit thermostabilem Vitamin B üben Baumwollsamenöl, Speck, Butter sowie Lebertran eine etwas verzögernde Wirkung aus, was besonders bei der Gruppe mit einer grossen Menge Vitamin A auffallend ist. Dies könnte daher rühren, dass Vitamin A und das thermostabile Vitamin B antagonistisch oder synergetisch wirken und dadurch den Stoffwechsel harmonisch erledigen.

Wenn auch unter den Gruppen mit dem Vitamin B-Komplex überhaupt keine Beriberisymptome auftreten, so werden sie doch hier bei der Hälfte der Ratten durch Baumwollsamenölgabe zusammen mit einer grossen Menge Vitamin A hervorgerufen, wie es bei den Gruppen ohne Vitamin A der Fall ist. Dies dürfte auf dem Fett oder Öl selbst und der Förderung der $\mathrm{B}_{1}$-Avitaminose durch Vitamin $\mathrm{A}$ beruhen. 
Wie aus Obigem ersichtlich, kann man auch bei diesen Versuchen Evans' u. Lepkovskys ,sparing action" nicht feststellen, sondern umgekehrt Förderung der $B_{1}-A$ vitaminose durch Fett oder $0 ̈ 1$ sowie Vitamin A und die antagonistische oder synergetische Wirkung des Vitamin B-Komplexes sowie des thermostabilen Vitamins B auf Fett oder Öl sowie Vitamin A.

Nur sieht man bei diesen Versuchen in der Gruppe mit Fett eine geringere Verzögerung der B-Avitaminose als in der Gruppe mit fettfreier Nahrung, was in gewissem Sinne für die Evans- und Lepkovskysche Theorie zu sprechen scheint.

\section{Schädigende Wirkung übermässigen Fetts.}

(a) Versuche ohne Vitamin A.

Bei den Gruppen mit Speck ist keine Schädigung des Fetts nachweisbar, dasselbe ist der Fall mit oder ohne Vitamin B-Faktoren; bei den Gruppen mit Baumwollsamenöl kann man im allgemeinen Schädigung durch Fett beobachten, und die Vitamin B-Faktoren spielen hier nicht die Rolle der Abwehr.

(b) Versuche mit Vitamin A.

Butter mit oder ohne Vitamin B-Faktoren schädigt nicht; Lebertran wirkt aber schädlich, und dabei kann man durch Zusatz des Vitamin B-Komplexes leichte Hemmung erzielen. Unter den Tieren mit Speck und Vitamin A in kleiner und mittelgrosser Menge wies mehr als die Hälfte der Tiere eine schädigende Wirkung auf, durch Zusatz von Vitamin B-Faktoren wirkte es weniger schädlich, während bei den Gruppen ohne und mit einer grossen Menge Vitamin A keine schädigende Wirkung auftrat. Es ist möglich, dass dies von dem Unterschied in der Ausnützung des Fetts durch Vitamin A abhängt.

Bei den mit Baumwollsamenöl gefütterten Gruppen (mit Vitamin A) trat immer schädigende Wirkung auf, durch Zusatz von Vitamin B-Faktoren (besonders des Vitamin B-Komplexes) wird sie vermindert.

Im allgemeinen sind harte Butter und Speck (von hohem Schmelzpunkt) öligem Lebertran und Baumwollsamenöl (von niedrigem Schmelzpunkt) überlegen; die Vitamin B-Faktoren hemmen bei Anwesenheit von Vitamin A die Schädigung durch Lebertran und Baumwollsamenöl. 


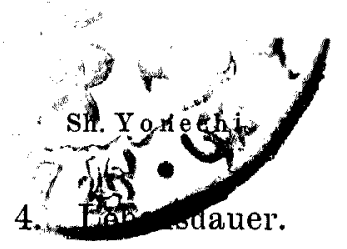

(a) Versuche ohne Vitamin A.

Bei den Gruppen mit Speck oder Baumwollsamenöl tritt frühzeitiger Tod ein, was der Schädigung durch Fett oder Öl selbst zuzuschreiben wäre. Thermostabiles Vitamin B übt eine verzögernde Wirkung aus, und bei den Gruppen mit dem Vitamin B-Komplex bleiben die meisten Tiere die ganze Versuchszeit hindurch am Leben.

Die Lebenserhaltung bei den Tieren mit Vitamin A-freiem Fett oder Öl ist nicht von der Art des Fetts, sondern von den Vitamin BFaktoren abhängig.

(b) Versuche mit Vitamin A.

Bei Lebertran-, Baumwollsamenöl- und Speckzusatz ohne Vitamin B-Faktoren ist die Lebensdauer der der Gruppe mit fettfreier Nahrung fast gleich, und bei Butterzusatz verlängert sie sich etwas.

Die Vitamin B-Faktoren, besonders der Vitamin B-Komplex, erhalten im allgemeinen bei Zusatz von Fett mit Vitamin A das Leben länger.

Dass Fett oder Öl erst zur Nahrung dient, wenn es Vitamin A enthält, ist schon allgemein bekannt. Anderseits bildet Fett oder Öl ein unentbehrliches Element der Nahrung. Welchen Einfluss übt denn fettfreie Nahrung auf Lebende aus?

Kürzlich haben $E v a n s$ u. Burr ${ }^{45}$ ) festgestellt, dass bei fettfreier Nahrung subnormales Wachstum eintritt; nach Burr u. Burr ${ }^{46}$ ) wird dabei Haarausfall in der Umgehung der Nase und des Mundes und der Augenlider, in der Kinmgegend und endlich am Rücken beobachtet, und dadurch werden die Symptome einer ArtDermatitis hervorgerufen, auch ödematöse hämorrhagische Anschwellung der Extremitäten kann vorkommen; am Schwanz wird eine spezifische Veränderung, der sogen. "scaly tail", dann Nekrose hervorgerufen, und Abmagerung sowie Atropie kommen vor. Burr u. Burri ${ }^{47}$ und Burr, Burr u. Mille $\mathrm{r}^{48}$ haben dann behauptet, dass zurBeseitigung dieser Mangelsymptome Fettsäuren wirksam sind. In Japan hat $\mathrm{T}$ ang $\mathrm{e}^{49}$ diese Behauptung nachgeprüft und sie bestätigt. Auch Sinclair ${ }^{50)}$ und Evans u. Lepk ov sk $\mathrm{y}^{51)^{52}}$ ) haben die Entstehung dieser Symptome angenommen und nachdrücklich behauptet, dass sie durch spezifische ungesättigte Fettsäure beseitigt werden können. Nach Hum e u. H. M. Sm i th ${ }^{53)}$ werden diese Symptome durch mechanische Wirkungen hervorgerufen, weil sie bei der Fütterung im kleinmaschigen Käfig niemals eintreten, und der „scaly tail" kann durch eine grosse Menge Hefe beseitigt werden. Demgegenüber hat T an $\mathrm{e}^{49}$ ) behauptet, dass Hefe solche Symptome nicht beseitigt. Welche Symptome treten denn umgekehrt bei übermässiger Fett- oder Ölfütterung auf? 
Nach All e $\mathrm{n}^{54}$ werden infolge übermässiger Fett- oder Ölfütterung Haarausfall, Körpergewichtsabnahme, terminale A taxie und allgemeiner Krampf hervorgerufen. Nach A. H. Smith u. Carry ${ }^{55}$ und $\mathrm{MacCl}$ ure u. Carr ${ }^{56}$ ) wirkt übermässige Lebertran- oder Baumwollsamenölfütterung trophisch ungünstig; Butter ist in dieser Hinsicht wohl etwas günstiger. Nach Friedberger u. Seidenberg, ${ }^{5 i}$ die wachsende Ratten untersucht haben, kleben bei der Fütterung mit übermässigem Fett oder Öl die Haare aneinander und werden büschelartig an Kopf, Bauch und in der Umgebung der Nase sowie des Mundes; dann tritt herdförmiger Haarausfall auf; die Haut wird kalt und dünn; Körpergewichtsabnahme und Atrophie treten auf; das Wachstum wird unterbrochen; frühzeitiger Tod tritt ein. Bei der Fütterung mit Olivenöl oder Lebertran sind diese Symptome auffallend; bei der Fütterung mit Speck oder Butter kommen sie ebenso vor, nur dass das Körpergewicht zunimmt und die Lebensdauer verlängert wird. Wenn man die Nahrung mit der Schlund sonde direkt in den Magen einführt, sind keine Dermatitissymptome nachweisbar. Auch nach Leibowitsch-Liwsehina, ${ }^{58}$ ) der seine Untersuchungen mit Olivenöl, Rizinusöl, Leinöl und Lebertran angestellt hat, werden bei allen Fällen nach einer Woche die Haare verklebt und erscheinen wie Büschel, und Haarausfall, Körpergewichtsverlust, trophische Störung und frühzeitiger Tod werden hervorgerufen. Bei der Fütterung mit Lebertran sind diese Erscheinungen am auffallendsten, bei Butter oder Speck aber nicht so deutlich. Also ist die Schädigung durch eine grosse Menge von Fett oder Öl von einer Reihe von Autoren anerkannt, wenn auch L evine u. A. H. Smith ${ }^{59}$ mitgeteilt haben, dass die Tiere auch bei Fütterung mit einer grossen Menge Lebertran oder Speck, ganz wie in der Norm, am Leben blieben.

Nach Berczelle $\left.{ }^{60}\right)$ fördert Fett oder öl ohne Vitamin A die Zersetzung der Körperfettgewebe. Nach Meska ${ }^{61)}$ rufen Fett- oder Ölarten ohne Vitamin A bei Menschen Seborrhoea hervor. Nach Ozaki ${ }^{62}$ hat von Vitamin A befreiter Lebertran keinen besonderen Nährwert, und in der Reihenfolge: Speck, Baumwollsamenöl, Lebertran, Butter, erhöht sich der Nährwert.

All dies zeigt übereinstimmend die Wichtigkeit des Vitamins A für den Fettstoff wechsel.

Hopkins, ${ }^{63}$ MacClure u. Carr, ${ }^{56}$ Matsumuro, Nakamura u. Ko$m a i^{(5)}$ und $\mathrm{Yamamot} 0^{(5) 66)}$ haben, im Gegensatz zu Obigem, als Folge der Fütterung von Vitamin A oder Lebertran in grosser Menge Schädignngen beobachtet, nämlich Haarverödung, Schleimhaut- und Hautentzündung, Abmagerung, Körpergewichtsverlust, Unterbrechung des Wachstums und frühzeitigen Tod.

Ich möchte auch einige Berichte über die Wechselbeziehungen zwischen Fett oder öl, Vitamin A, Vitamin B-Komplex und anderen Vitamin hervorheben.

Nach Frank ${ }^{6 \ni}$ ) kann die oben erwähnte schädigende Wirkung übermässigen Fetts durch Vitamin B und Vitamin C zusammen, auch durch den Vitamin B-Komplex allein, aber niemals durch Vitamin $\mathrm{C}$ allein beseitigt werden. Auch nach Éderer ${ }^{(8)}$ ist übermässige Fettfütterung olne den Vitamin B-Komplex für die Lebensbedingungen wohl ungünstig. 
Nach N orris und Church ${ }^{(9)}$ werden durch Fütterung einer grossen Lebertrammenge und durch langdauernde Fütterung von Isoamylamin und Cholin, als Bestandteile des Lebertrans, Paralyse, Krämpfe und Wachstumsstillstand hervorgernfen. Sie behaupten, dass diese Erscheinungen auf die Toxizität des Lebertrans zurückzuführen seien und zugleich vom Vitamin B-KomplexGehalt in der Grundnahrung bestimmt würden, und dass eine grosse Menge des Vitamin B-Komplexes eine antagonistische Wirkung auf die Toxizität des Vitamins A ausüben könne. Yamamoto ${ }^{65) 66)}$ ist der Meinung, dass man die Schädigung des Lebertrans verhältnismässig gut durch Hefegabe beseitigen kann.

Aus obiger Übersicht der Literatur wird man eine auffallende Übereinstimmung der durch fettfreie Nahrung und der durch übermässige Fettgabe hervorgerufenen Symptome erkennen. Beiden gemeinsam sind verstärkte Neigung zur Schleimhaut- und Hautentzünđung; Verödung, Verklebung und Verbüschelung der Haare; Haarausfall, Körpergewichtsverlust, Wachstumsstillstand und frühzeitiger Tod.

\section{Zusammenfassung des zweiten Teils.}

1. Auf die Körpergewichtserhaltung wirkt Fett oder Öl in grosser Menge schädlich, wenn es auch je nach diesem oder jenem einen gewissen Unterschied dabei gibt; sie ist jedenfalls ganz unabhängig vom Vitamin A-Gehalt. Thermostabiles Vitamin B und der Vitamin B-Komplex wirken antagonistisch oder synergetisch auf die durch übermässiges Fett oder Öl eingetretene Abmagerung.

2. Durch übermässige Fett- oder Ỏlmengen treten schädigende Wirkungen auf, d. h. Körpergewichtsverlust, Abmagerung u. a. wodurch wiederum die Entstehung der Beriberisymptome gefördert wird, wenn auch je nach der Art des Fetts dabei Unterschiede bestehen. Bei den mit Speck- und Baumwollsainenöl, zusammen mit Vitamin A, gefütterten Tieren tritt, je grösser die Vitamin A-Menge ist, umso früher B-Avitaminose ein; deshalb wirkt Vitamin A auf die B-Avitaminose fördelnd.

3. Die schädigende Wirkung übermässigen Fetts steht zu dem in Fett oder Öl enthaltenen Vitamin A-Gehalt in keiner Beziehung. Inbezug auf die schädigende Wirkung uibermässigen Fett hat festes Fett (von hohem Schmelzpunkt), z. B. Butter, im Vergleich mit Öl (von niedrigem Schmelzpunkt), z. B. Lebertran, ausgezeichnete Eigenschaften. Bei direkter Einführung des Öls in den Magen von Tauben trat Schädigung des Fetts ein. Deshalb darf man dies nicht, wie Friedberger, unbedingt auf die äussere Berühung zurückführen, sondern muss es, wie schon oben erwähnt, vielmehr der Toxität von 
Fett oder Öl zuschreiben. Thermostabiles Vitamin B und der Vitamin B-Komplex hemmen den Schaden durch übermässige Fettfütterung.

4. Auf die Lebensdauer wirkt eine iibermässige Fett- oder Ölmenge verkürzend und der Vitamin B-Komplex und das thermostabile Vitamin $B$ verlängernd.

5. Im übrigen konnte ich bei der Fütterung mit fettfreier Nahrung nicht, wie Burr und Burr, Tange u. a. bei ihren Versuchen, deutliche Veränderungen beobachten. Ob der Grund dafür in der Nichtentfettung der nur in sehr geringer Menge genommenen Hefe liegt, bleibt freilich noch unklar. Jedoch habe ich dabei Verklebung der Haare und frühzeitigen Tod beobachtet. Wenn auch der Schwanz kein "scaly tail" wurde, so habe ich doch ,dandruff" des Scliwanzes beobachtet. Demgegenüber bestand bei Gabe von $0,2 \mathrm{~g}$ Hefe kein besonderer Ünterschied.

\section{Schluss.}

Aus obigen Versuchsergebnissen kann man folgende Schlüsse ziehen.

(1) Angenommen, dass auf die Schädigung durch übermässiges Fett und die $\mathrm{B}_{1}$-Avitaminose übermässige Fett- oder Vitamin A-Gabe nur im oben erörterten Sinne fördernd wirkt, so kann man sagen, dass der Vitamin B-Komplex und das thermostabile Vitamin B diese Störung beseitigen, d. h. antagonistisch wirken; also üben Fett, Vitamin A und Vitamin B-Fakturen eine synergetische oder regulierende Wirkung auf den ganzen Stoffwechsel bei Lebenden aus.

(2) Welln man bei verschiedenen Krankheiten zur Diättherapie Fett oder Öl und Vitamin A benutzen will, so muss man auch die Art des Fetts berücksichtigen (z. B. besser Butter oder Speck als Lebertran oder Baumwollsamenöl) und ferner genügend Vitamin B-Komplex geben.

\section{Literatur.}

(1) Evans u. Lepkovskg, Jl. of Biol. Chem., 1929, 83, 269.

(2) Evansu. Lepkovsky, Jl. of Biol. Chem., 1932, 96, 165.

(3) Evans u. Le pkovsky, J1, of Biol. Chem., 1932, 96, 179.

(4) Evans u. Lepkovsky, J1. of Biol. Chem., 1932, 99, 235. 83, 269.

(5) MacCollum, zit. nach Evans u. Lepkovsky, Jl. of Biol. Chem., 1929,

(6) Saiki, Higuchi, Kondo u. Matsuzawa, The Chemical Analysis of Food in Japan (jap.), Tokyo 1931 . 
(7) Matsuoka, Die Chremie der Ernährung (jap.), Tokyo 1933, 45-50.

(8) Fu.jimaki u. A rimoto, Die Chemie von Ernährung und Nahrungsmittel (jap.), Tokyo 1931, 400.

(9) M. Miu ra, Bulletin of the Institute of Physical and Chemical Research (jap.), $1926,5,525$.

(10) Sch all, Nahrungsmitteltabelle, Leipzig 1929, 100.

(11) A bderhalden, Münch. med. Wschr., 1933, 80, 722.

(12) A bderhalden n. Wertheimer, Pflüger's Archiv, 1932, 230, 601.

(13) A s ad a, Biochem. Ztschr., 1923, 139, 234.

(14) Bickel, Biochem. Ztschr., 1924, 146, 493.

(15) Bickel u. Ka ufmann-Cosla, Münch. med. Wschr., 1925, 72, 1018.

(16) Tscherkes, Biochem, Ztschr., 1926, 167, 203.

(17) Y a ma a a, Shakwai Igaku Zasshi, 1931, Nr. 532, Nr. 533, 249, 366.

(18) Koll a t, Naturwissensehaft, 1933, 21, 537.

(19) F u n k, Jl. of Physiology, 1919-20, 53, 247.

(20) Collazo u. Pi-S uner Bay o, Biochem. Ztschr., 1931, 238, 335.

(21) Kaufmann-Cosla, Vasilico u. Örin, Arch. f. exp. Pathol. u. Pharn., $1932,164,608$.

(22) In o ue, Fukuoka Ikadaigaku Zasshi, 1933, 26, 217.

(23) Matsuoka, Referat der in der 23ten Versammelung gehaltenen Vorträge von Rikwagaku-Kenkyu-Sho (jap.), 1933.

(24) Funk and Dabin, Science, 1920, 52, 447.

(25) A bder'halden, Pflïger's Archiv, 1923, 198, 571.

(26) O s born u. Mendel, Jl. of Biol. Chem., 1922, 54, 739.

(27) A d dis, MacK a y u. M a c K a y, Jl. of Biol. Chem., 1926-27, 71, 139.

(28) Osborn, Mendel, Park, u. Winternitz, Jl. of Biol. Chem., 1926-27, 71, 317 .

(29) Francis, S m ith u. Mo ise, Proc. Soc. for Biol. and Med., 1928-29, 26, 725.

(30) T s cherkes, Biochem. Ztschr., 1922, 133, 75.

(31) Reader u. Drummond, Biochem. Jl. 1926, 20, 1256.

(32) Hassan u. Drummond, Biochem. Jl. 1927, 21, 653.

(33) Plimmer u. a., Biochem. Jl., 1927, 21, 913.

(34) Hartwell, Biochem. Jl. 1927, 21, 1076.

(35) Francis, Smith u. Mendel, Jl. of Nutrition, 1983, 6, 493.

(36) Pru.nty u. Roscoe, Biochem. Jl., 1933, 27, 699.

(37) Willi a ms u. Wa termann, Proc. Soc. for Biol. and Med., 1927, 25, 1.

(38) Willi a ms u. Water'm a n n, Jl, of Biol. Chem., 1928, 78, 311.

(39) Peter's, zit. nach Levis, Jl. of Nutrition, 1933, 6, 559.

(40) Peters, Carter u. Kinesley, Biochein. Jl. 1930, 24, 1832, 1844.

(41) György, Kuhnu. Wagner-J a uregg, Kl. Wschr., 1933, 12, 1241.

(42) Reader, Biochem. J1., 1929, 23, 689.

(43) Rea der, Biochem. Jl., 1930, 24, 1827.

(44) Halliday, N un n u. Fis cher, Jl. of Biol. Chem., 1932, 96, 479.

(45) Evans u. Burr, Proc. Soc. for Exp. Biol. and Med., 1927-28, 25, 390.

(46) Burru. Burr, Jl. of Biol. Chem., 1929, 82, 345.

(47) Burr a. Burr, Jl. of Biol. Chem., 1930, 86, 587.

(48) Burr, Burr, u. Miller, Jl. of Biol. Chem, 1932, 97, 1.

(49) Tange, Scientific Papers of the Institute of Physical and Chemical Researcb 1932, 20, 13.

(50) S incla ir, Proc. Soc. for Exp. Biol. and Med., 1929-30, 27, 1059. 
(51) Evansu. Lepkovsky, Jl. of Biol. Chem., 1932, 96, 143, 157.

(52) Evans u. Lepkovsky, Jl. of Biol. Chem., 1932, 99, 231, 237.

(53) H $\mathrm{m} \mathrm{m}$ e u. H. H. S m it h, Biochem. Jl., 1931, 25, 300.

(54) Alle n, Am. Jl. of Physiol., 1916-17, 42, 583.

(55) A. H. S mith u. Carry, Jl. of Biol. Chem., 1923-24, 58, 425.

(56) MacClure u. Carr, Am. Jl. of Physiol., 1925, 74, 70.

(57) Friedberger u. Seiden berg, Biochem. Ztschr., 1928, 200, 289.

(58) Leibowitsch-Liwschina, Biochem. Ztschr., 1931, 231, 260.

(59) Levine u. S mith, Jl. of Biol. Chem., 1927, 72, 223.

(60) Berczeller, Biochem. Ztschr., 1924, 129, 217.

(61) Mesk a, Dermat. Ztschr., 1924, 78, 552.

(62) $0 \mathrm{zaki}$, Jl. of the Agricultural Chemical Society of Japan (jap.) 1926, 3, 120.

(63) Ho pkins, Brit. Med. Jl., 1923, 2, 691.

(64) Matsumuro, Nakamura, u. Komai, Report of the Imperial Government Institute for Nutrition, 1933, 4, 77.

(65) Y a m a moto, Referat der in der 23ten Versammelung gehaltenen Vorträge von Rikwagaku-Kenkyu-Sho (jap.), 1933.

(66) Y a ma moto, Bulletin of Physical and Chemical Research, 1934, 13, 1.

(67) Frank, Monatsschrift f. Kinderheilkunde, 1923, 25, 147.

(68) Éderer, Biochem. Ztschr., 1925, 158, 197.

(69) Noris u. Church, Jl. of Biol. Chem., 1930, 89, 437. 